\title{
Controlled Lagrangian Systems with Gyroscopic Forcing and Dissipation
}

\author{
Craig Woolsey * \\ Aerospace \& Ocean Engineering \\ Virginia Tech \\ Blacksburg, VA 24061 \\ cwoolsey@vt.edu \\ Anthony M. Bloch ${ }^{\dagger}$ \\ Department of Mathematics \\ University of Michigan \\ Ann Arbor, MI 48109 \\ abloch@umich.edu \\ Naomi Ehrich Leonard $\ddagger$ \\ Mechanical \& Aerospace Engineering \\ Princeton University \\ Princeton, NJ 08544 \\ naomi@princeton.edu
}

\author{
Chevva Konda Reddy* \\ Engineering Science \& Mechanics \\ Virginia Tech \\ Blacksburg, VA 24061 \\ konda@vt.edu \\ Dong Eui Chang \\ CAS, ENSMP \\ 60, bd. Saint-Michel \\ 75006, Paris, FRANCE \\ dchang@cas.ensmp.fr \\ Jerrold E. Marsden § \\ Control \& Dynamical Systems 107-81 \\ California Institute of Technology \\ Pasadena, CA 91125 \\ marsden@cds.caltech.edu
}

European Journal of Control, 10, number 5, 2004

\begin{abstract}
This paper describes a procedure for incorporating artificial gyroscopic forces in the method of controlled Lagrangians. These energy-conserving forces provide additional freedom to expand the basin of stability and tune closed-loop system performance. We also study the effect of physical dissipation on the closed-loop dynamics and discuss conditions for stability in the presence of natural damping. We apply the technique to the inverted pendulum on a cart, a case study from previous papers. We develop a controller which asymptotically stabilizes the inverted equilibrium at a specific cart position for the conservative dynamic model. The region of attraction contains all states for which the pendulum is elevated above the horizontal plane. We also develop conditions for asymptotic stability in the presence of linear damping.
\end{abstract}

\section{Introduction}

The method of controlled Lagrangians is a constructive technique for stabilizing underactuated mechanical systems. The method has roots in [8], where it was shown that an internal rotor can be used to effectively

\footnotetext{
${ }^{*}$ Research partially supported by NSF grant CMS-0133210 and ONR grant N00014-01-1-0588.

${ }^{\dagger}$ Research partially supported by NSF grants DMS 0103895 and 0305837 and AFOSR.

${ }^{\ddagger}$ Research partially supported by NSF grant CCR-9980058, ONR grants N00014-02-1-0826 and N00014-02-1-0861 and AFOSR grant F49620-01-1-0382.

${ }^{\S}$ Research partially supported by the California Institute of Technology and ONR Contract N00014-02-1-0826
} 
shape the kinetic energy of a spacecraft in order to stabilize steady rotation about the intermediate principal axis of inertia. As initially developed by Bloch, Leonard, \& Marsden [9, 10, 13], the method of controlled Lagrangians provides a kinetic-shaping control design tool for a class of underactuated mechanical systems which exhibit an abelian Lie group symmetry in the input directions. This class includes the spacecraft example described above and the classic inverted pendulum on a controlled cart. Later work on controlled Lagrangians $[6,11]$ introduced additional control freedom by allowing potential shaping and by relaxing the symmetry condition on the original and feedback-modified potential energy. Still more control freedom may be introduced by allowing for generalized gyroscopic forces in the closed-loop dynamics. Gyroscopic control forces predate the method of controlled Lagrangians, appearing in the work of [28] and later in the developments of [7]. In related earlier work in [27], Poisson control systems were introduced and studied.

Concurrent with the development of the method of controlled Lagrangians, others have explored kinetic shaping in more general settings. A very reasonable question is: What are the general conditions under which a feedback-controlled, underactuated mechanical system is Lagrangian or Hamiltonian? That is, without imposing any a priori requirements on an underactuated mechanical system, when is it possible to choose feedback such that the closed-loop equations are Lagrangian or Hamiltonian? This question was addressed in [2] and [20] on the Lagrangian side and in [4] and [25] on the Hamiltonian side. The question is one of feedback equivalence; one obtains conditions in the form of partial differential equations.

Chang [17] investigated the use of artificial gyroscopic forces to enhance the controlled Lagrangian design process in some depth. An example, involving stabilization of an inverted pendulum on a rotor arm, illustrated the utility of this idea. In [18], the appearance of generalized gyroscopic forces in the controlled Lagrangian dynamics was related explicitly to a modification of the dynamic structure in the Hamiltonian setting. It was also shown in [18] that the most general formulation of the method of controlled Lagrangians, in which one places no prior restrictions on the class of eligible mechanical systems, is equivalent to the interconnection and damping assignment, passivity based control (IDA-PBC) technique described in [4] and $[25]$.

While it is important and worthwhile to understand feedback equivalence of general Lagrangian and Hamiltonian systems, there are advantages in restricting one's view to a smaller class of systems. For example, the control design procedure defined in [13] is algorithmic and does not rely on case-by-case solution of a set of PDEs. In this paper, we continue in this spirit and restrict the form of kinetic energy modifications to that described in [13]. We also allow forces due to a modified potential energy in the closed-loop dynamics, as in [6], as well as artificial gyroscopic forces. The approach strikes a physically motivated balance between the algorithmic simplicity of the method described in $[6,13]$ and the elegance and generality of [18].

We apply the technique to the inverted pendulum on a cart system, a case study from previous papers. A feedback control law is derived which makes the desired equilibrium a strict minimum of the control-modified energy. In the absence of physical damping, the control law provides stability in a basin that includes all states for which the pendulum is elevated above the horizontal plane. The addition of feedback dissipation provides asymptotic stability within this same stability basin. This region of attraction is larger than any given earlier by the method of controlled Lagrangians. Two features distinguish the present example from our previous results and those of others, such as Acosta, Ortega, \& Astolfi [1], Auckly, Kapitanski, \& White [2], and Hamberg [20]. First, the closed-loop Lagrangian system includes artificial gyroscopic forces. Second, we consider the issue of physical damping in detail and study its effect on the closed-loop dynamics.

Modifying kinetic energy through feedback affects the manner in which damping enters the system. The problem is well-illustrated by the cart-pendulum example. Even though the desired equilibrium is a strict minimum of the control-modified energy, simple Rayleigh dissipation makes the closed-loop system unstable. Careful analysis shows that asymptotic stability may be recovered through an appropriate choice of feedback dissipation, however it is not "automatic." Working in the Hamiltonian setting, Gómez-Estern \& van der Schaft [19] suggest procedures for recovering asymptotic stability in systems with feedback-modified kinetic 
energy. An essential requirement is that the system satisfy a dissipation condition involving the original and modified kinetic metrics and a matrix of damping coefficients. As an alternative to this type of controller re-design, one may attempt to address the issue of damping during the matching process, as in [3].

The controller presented here has been implemented experimentally [26]. For the experimental apparatus, the damping of the pendulum motion is well-modeled as linear in angular rate. The damping of the cart's motion, however, follows a nonlinear friction model. Static friction degrades the energy-shaping controller's local performance by inducing limit cycle oscillations. A well-designed linear state feedback control law, on the other hand, eliminates these oscillations. To recover the best features of both controllers (the large region of attraction and good local performance), a switching control law was successfully implemented. These experimental results further illustrate the importance of considering physical dissipation in systems whose kinetic energy has been modified through feedback.

Section 2 reviews details of the method of controlled Lagrangians as it was originally presented in $[6,13]$. In particular, this section defines the form of the closed-loop kinetic energy metric, a form inspired by geometric mechanics. Section 3 describes how to include artificial gyroscopic forces and presents a general procedure for "matching" the original dynamic equations with the closed-loop equations. Section 4 explains how additional external forces, such as physical damping or feedback dissipation, affect the control-modified energy. Section 5 describes control design and stability analysis for the example of a pendulum on a cart. We discuss conclusions and future directions in Section 6.

\section{Review of the Controlled Lagrangian Formulation}

This section provides a brief introduction to the method of controlled Lagrangians. One begins with a Lagrangian mechanical system with Lagrangian $L=K-V$, where $K$ is the uncontrolled kinetic energy and $V$ is the uncontrolled potential energy. We modify both kinetic and potential energy to produce a new controlled Lagrangian. We also allow generalized gyroscopic forces in the closed-loop system; these correspond to a modified dynamic structure when one passes to the Hamiltonian setting [18].

The Setting. Suppose the system has configuration space $Q$ and that an abelian Lie group $G$ acts freely and properly on $Q$. (See [22] or [23] for a brief discussion of Lie groups and group actions.) The goal of kinetic shaping is to control the variables lying in the shape, or orbit, space $S=Q / G$ using controls that act directly on the variables lying in $G$. This approach differs from the idea of locomotion via shape change, where one controls the shape variables in order to effect group motions; see [5] or [16], for example.

Assume that $K: T Q \rightarrow \mathbb{R}$ is invariant under the given action of $G$ on $Q$. The modification of $L$ involves changing the metric tensor $g(\cdot, \cdot)$ that defines the kinetic energy $K=\frac{1}{2} g(\dot{q}, \dot{q})$. The tangent bundle $T Q$ can be split into a sum of horizontal and vertical parts defined as follows: for each tangent vector $\mathbf{v}_{q}$ at a point $q \in Q$, we can write a unique decomposition

$$
\mathbf{v}_{q}=\operatorname{Hor} \mathbf{v}_{q}+\operatorname{Ver} \mathbf{v}_{q},
$$

such that the vertical part is tangent to the orbit of the $G$-action and the horizontal part is the metric orthogonal to the vertical space; that is, it is uniquely defined by requiring that

$$
g\left(\mathbf{v}_{q}, \mathbf{w}_{q}\right)=g\left(\operatorname{Hor} \mathbf{v}_{q}, \operatorname{Hor} \mathbf{w}_{q}\right)+g\left(\operatorname{Ver} \mathbf{v}_{q}, \operatorname{Ver} \mathbf{w}_{q}\right) .
$$

for every $\mathbf{v}_{q}, \mathbf{w}_{q} \in T_{q} Q$ and that $\operatorname{Ver} \mathbf{v}_{q}$ and $\operatorname{Ver} \mathbf{w}_{q}$ are vertical. Figure 1 depicts the decomposition of $T_{q} Q$ into the horizontal space $\operatorname{Hor}_{q}$ and the vertical space $\operatorname{Ver}_{q}$. Define local coordinates $x^{\alpha}$ for $Q / G$ and $\theta^{a}$ for $G$. (In examples, the latter coordinates are often cyclic; hence the notation $\theta^{a}$.) The kinetic energy may be written as

$$
K=\frac{1}{2} g_{\alpha \beta} \dot{x}^{\alpha} \dot{x}^{\beta}+g_{\alpha b} \dot{x}^{\alpha} \dot{\theta}^{b}+\frac{1}{2} g_{a b} \dot{\theta}^{a} \dot{\theta}^{b},
$$


Figure 1: Decomposition of $T_{q} Q$.

where $g_{\alpha \beta}, g_{\alpha b}$, and $g_{a b}$ are the local components of $g(\cdot, \cdot)$. Requirement (1) may be thought of as a block diagonalization procedure or "completing the square," since it suggests rewriting the kinetic energy in the form

$$
K=\frac{1}{2}\left(g_{\alpha \beta}-g_{\alpha a} g^{a b} g_{b \beta}\right) \dot{x}^{\alpha} \dot{x}^{\beta}+\frac{1}{2} g_{a b}\left(\dot{\theta}^{a}+g^{a c} g_{c \alpha} \dot{x}^{\alpha}\right)\left(\dot{\theta}^{b}+g^{b d} g_{d \beta} \dot{x}^{\beta}\right) .
$$

(Note: If $\left[g_{a b}\right]$ represents the matrix form of the tensor $g_{a b}$ then $\left[g^{a b}\right]$ is the matrix inverse of $\left[g_{a b}\right]$.) Let the pair $\left(\dot{x}^{\alpha}, \dot{\theta}^{a}\right)$ represent the local expression for velocity $\mathbf{v}_{q}$. Then

$$
\begin{aligned}
\operatorname{Ver} \mathbf{v}_{q} & =\left(0, \dot{\theta}^{a}+g^{a b} g_{\alpha b} \dot{x}^{\alpha}\right) \\
\operatorname{Hor} \mathbf{v}_{q} & =\left(\dot{x}^{\alpha},-g^{a b} g_{\alpha b} \dot{x}^{\alpha}\right) .
\end{aligned}
$$

The given choice of horizontal space coincides with that given by the mechanical connection. Let $\mathfrak{g}$ denote the Lie algebra of $G$. The mechanical connection $\zeta$ is a Lie algebra-valued, vertical one-form, i.e., a map from $T Q$ to $\mathfrak{g}$ which annihilates the horizontal component of velocity,

$$
\zeta\left(\mathbf{v}_{q}\right)=\zeta\left(\operatorname{Ver} \mathbf{v}_{q}\right) \in \mathfrak{g} .
$$

Let $\xi_{Q}$ denote the infinitesimal generator corresponding to an element $\xi \in \mathfrak{g}$. For each $\xi \in \mathfrak{g}, \xi_{Q}$ is a vector field on the configuration manifold $Q$ and its value at a point $q \in Q$ is denoted $\xi_{Q}(q)$. In coordinates, the infinitesimal generator $\left[\zeta\left(\mathbf{v}_{q}\right)\right]_{Q}(q) \in T_{q} Q$ corresponds to $\left(0, g^{a b} g_{\alpha b} \dot{x}^{\alpha}\right)$. This term appears as the "velocity shift" in the expressions (3) and (4). For a thorough discussion of the mechanical connection, and its relationship to more general connection forms, see [5].

The Controlled Lagrangian. An essential feature of the controlled Lagrangian is the feedback-modified kinetic energy. The modification consists of three ingredients:

1. a different choice of horizontal space, denoted $\mathrm{Hor}_{\tau}$,

2. a change $g \rightarrow g_{\sigma}$ of the metric acting on horizontal vectors, and

3. a change $g \rightarrow g_{\rho}$ of the metric acting on vertical vectors.

The first ingredient above can be thought of as a shift in the mechanical connection. Let $\tau$ be a Lie algebravalued, $G$-equivariant horizontal one-form on $Q$; that is, a one-form with values in the Lie algebra $\mathfrak{g}$ of $G$ 
that annihilates vertical vectors. ${ }^{1}$ The $\tau$-horizontal space at $q \in Q$ consists of tangent vectors to $Q$ at $q$ of the form $\operatorname{Hor}_{\tau} \mathbf{v}_{q}=\operatorname{Hor}_{q}-[\tau(\mathbf{v})]_{Q}(q)$, which also defines the $\tau$-horizontal projection $\mathbf{v}_{q} \mapsto$ Hor $_{\tau} \mathbf{v}_{q}$. The $\tau$-vertical projection operator is defined by $\operatorname{Ver}_{\tau} \mathbf{v}_{q}=\operatorname{Ver} \mathbf{v}_{q}+[\tau(\mathbf{v})]_{Q}(q)$. The left half of Figure 2 depicts the role of $\zeta$ in the original decomposition; the right half depicts the analogous role of $\tau$ in defining the new decomposition.

Figure 2: Original (left) and modified (right) decompositions of $T_{q} Q$.

Given $\tau, g_{\sigma}$, and $g_{\rho}$, define the control-modified kinetic energy

$$
K_{\tau, \sigma, \rho}(\mathbf{v})=\frac{1}{2}\left[g_{\sigma}\left(\operatorname{Hor}_{\tau} \mathbf{v}_{q}, \operatorname{Hor}_{\tau} \mathbf{v}_{q}\right)+g_{\rho}\left(\operatorname{Ver}_{\tau} \mathbf{v}_{q}, \operatorname{Ver}_{\tau} \mathbf{v}_{q}\right)\right] .
$$

Assume that $g=g_{\sigma}$ on Hor and that Hor and Ver are orthogonal for $g_{\sigma}$. Then, as shown in [13],

$$
K_{\tau, \sigma, \rho}(\mathbf{v})=K\left(\mathbf{v}+\tau(\mathbf{v})_{Q}\right)+\frac{1}{2} g_{\sigma}\left(\tau(\mathbf{v})_{Q}, \tau(\mathbf{v})_{Q}\right)+\frac{1}{2}\left(g_{\rho}-g\right)\left(\operatorname{Ver}_{\tau}(\mathbf{v}), \operatorname{Ver}_{\tau}(\mathbf{v})\right) .
$$

The controlled Lagrangian is

$$
L_{\tau, \sigma, \rho}(\mathbf{v})=K_{\tau, \sigma, \rho}(\mathbf{v})-(V(q)+\tilde{V}(q))
$$

where $\tilde{V}(q)$ is an artificial potential function which modifies the effective potential energy in the closed-loop system. We define $V^{\prime}(q)=V(q)+\tilde{V}(q)$ to be the complete, control-modified potential energy. Then, using (5),

$$
L_{\tau, \sigma, \rho}(\mathbf{v})=K\left(\mathbf{v}+\tau(\mathbf{v})_{Q}\right)+\frac{1}{2} g_{\sigma}\left(\tau(\mathbf{v})_{Q}, \tau(\mathbf{v})_{Q}\right)+\frac{1}{2}\left(g_{\rho}-g\right)\left(\operatorname{Ver}_{\tau}(\mathbf{v}), \operatorname{Ver}_{\tau}(\mathbf{v})\right)-V^{\prime}(q) .
$$

The goal is to determine conditions on the original kinetic energy, on the energy modification parameters $\left(\tau, g_{\sigma}\right.$, and $\left.g_{\rho}\right)$, and on the potential functions $V$ and $\tilde{V}$ such that a particular choice of feedback yields closed-loop equations which are Euler-Lagrange equations for $L_{\tau, \sigma, \rho}$. These conditions are referred to as "matching" conditions. They ensure that no inputs are necessary in uncontrolled directions in order to effect the desired closed-loop dynamics. In examples, the matching conditions often leave freedom in the control parameters which can be used to satisfy conditions for stability.

\footnotetext{
${ }^{1}$ One of the basic requirements of a connection is that, when applied to an infinitesimal generator of the group at any point $q \in Q$ (say $\xi_{Q}(q) \in T_{q} \operatorname{Orb}(q)$ ) one recovers the Lie algebra element $(\xi)$. For this condition to be maintained when $\tau$ is added to $\zeta$, the one-form $\tau$ must be horizontal; this means that it should vanish when applied to vertical vectors which are vectors of the form $\xi_{Q}(q)$.
} 


\section{Matching}

Assume that the Euler-Lagrange equations hold for a mechanical system with Lagrangian

$$
L\left(x^{\alpha}, \theta^{a}, \dot{x}^{\alpha}, \dot{\theta}^{a}\right)=\frac{1}{2} g_{\alpha \beta} \dot{x}^{\alpha} \dot{x}^{\beta}+g_{\alpha b} \dot{x}^{\alpha} \dot{\theta}^{b}+\frac{1}{2} g_{a b} \dot{\theta}^{a} \dot{\theta}^{b}-V\left(x^{\alpha}, \theta^{a}\right) .
$$

The control effort $u_{a}$ enters in the $\theta^{a}$ direction so that the open-loop equations of motion are

$$
\begin{gathered}
\frac{d}{d t} \frac{\partial L}{\partial \dot{x}^{\alpha}}-\frac{\partial L}{\partial x^{\alpha}}=0 \\
\frac{d}{d t} \frac{\partial L}{\partial \dot{\theta}^{a}}-\frac{\partial L}{\partial \theta^{a}}=u_{a} .
\end{gathered}
$$

Suppose we wish to stabilize an unstable equilibrium

$$
\left(x^{\alpha}, \theta^{a}, \dot{x}^{\alpha}, \dot{\theta}^{a}\right)_{e}=\left(x_{e}^{\alpha}, \theta_{e}^{a}, 0,0\right)
$$

for the uncontrolled system (8). ${ }^{2}$ The method of controlled Lagrangians provides, under certain conditions, a control law $u_{a}$ for which the closed-loop equations are Lagrangian with respect to a modified Lagrangian $L_{\tau, \sigma, \rho}\left(x^{\alpha}, \theta^{a}, \dot{x}^{\alpha}, \dot{\theta}^{a}\right)$. In prior treatments, the closed-loop Euler-Lagrange equations included no generalized forces. More generally, one may seek conditions under which

$$
\begin{aligned}
\frac{d}{d t} \frac{\partial L_{\tau, \sigma, \rho}}{\partial \dot{x}^{\alpha}}-\frac{\partial L_{\tau, \sigma, \rho}}{\partial x^{\alpha}} & =\mathcal{S}_{\alpha \beta} \dot{x}^{\beta}+\mathcal{S}_{\alpha b} \dot{\theta}^{b} \\
\frac{d}{d t} \frac{\partial L_{\tau, \sigma, \rho}}{\partial \dot{\theta}^{a}}-\frac{\partial L_{\tau, \sigma, \rho}}{\partial \theta^{a}} & =\mathcal{S}_{a \beta} \dot{x}^{\beta}+\mathcal{S}_{a b} \dot{\theta}^{b}
\end{aligned}
$$

where the terms $\mathcal{S}_{i j}$ are the local components of a velocity-dependent two-form $\mathcal{S}$. In coordinates, $\mathcal{S}$ is a skew-symmetric matrix whose components depend on both the configuration and the velocity. More formally, $\mathcal{S} \in \Gamma\left(T Q, T^{*} Q \wedge T^{*} Q\right)$; a more thorough discussion appears in [17], including the special case where $\mathcal{S}$ defines a closed two-form.

Skew-symmetry of $\mathcal{S}$ ensures that the control modified energy corresponding to $L_{\tau, \sigma, \rho}$ is conserved; the corresponding generalized forces are referred to as "gyroscopic." The conditions under which equations (10) hold are the "matching conditions." These conditions ensure that equations (10) require no control authority in unactuated directions.

Let

$$
\zeta_{\alpha}^{a}=g_{\alpha c} g^{c a} \quad \text { and } \quad \tau_{\alpha}^{a}
$$

represent the coordinate expressions for the mechanical connection and the $\tau$-parameterized modification to this connection, respectively, as depicted in Figure 2. Also, let $\sigma_{a b}$ and $\rho_{a b}$ represent the "ab" components of $g_{\sigma}$ and $g_{\rho}$, respectively. Then, in coordinates, the controlled Lagrangian becomes

$$
\begin{aligned}
L_{\tau, \sigma, \rho}\left(x^{\alpha}, \theta^{a}, \dot{x}^{\alpha}, \dot{\theta}^{a}\right)= & L\left(x^{\alpha}, \theta^{a}, \dot{x}^{\alpha}, \dot{\theta}^{a}+\tau_{\alpha}^{a} \dot{x}^{\alpha}\right)+\frac{1}{2} \sigma_{a b} \tau_{\alpha}^{a} \tau_{\beta}^{b} \dot{x}^{\alpha} \dot{x}^{\beta} \\
& +\frac{1}{2}\left(\rho_{a b}-g_{a b}\right)\left(\dot{\theta}^{a}+\left(g^{a c} g_{c \alpha}+\tau_{\alpha}^{a}\right) \dot{x}^{\alpha}\right)\left(\dot{\theta}^{b}+\left(g^{b d} g_{d \beta}+\tau_{\beta}^{b}\right) \dot{x}^{\beta}\right)-\tilde{V}\left(x^{\alpha}, \theta^{a}\right) \\
= & \frac{1}{2}\left(g_{\tau, \sigma, \rho}\right)_{\alpha \beta} \dot{x}^{\alpha} \dot{x}^{\beta}+\left(g_{\tau, \sigma, \rho}\right)_{\alpha b} \dot{x}^{\alpha} \dot{\theta}^{b}+\frac{1}{2}\left(g_{\tau, \sigma, \rho}\right)_{a b} \dot{\theta}^{a} \dot{\theta}^{b}-V^{\prime}\left(x^{\alpha}, \theta^{a}\right) .
\end{aligned}
$$

\footnotetext{
${ }^{2}$ As described in [13], in cases where $\theta^{a}$ is cyclic, one may instead stabilize steady motions of the form
}

$$
\left(x^{\alpha}, \dot{x}^{\alpha}, \dot{\theta}^{a}\right)_{e}=\left(x_{e}^{\alpha}, 0, \dot{\theta}_{e}^{a}\right) .
$$


Equation (11) introduces the coordinate form of the modified kinetic energy metric $g_{\tau, \sigma, \rho}$. Let

$$
B_{\alpha \beta}=g_{\alpha \beta}-g_{\alpha a} g^{a b} g_{b \beta}
$$

and let

$$
A_{\alpha \beta}=B_{\alpha \beta}+\tau_{\alpha}^{c} \sigma_{c d} \tau_{\beta}^{d}
$$

It is readily shown that

$$
\begin{aligned}
\left(g_{\tau, \sigma, \rho}\right)_{\alpha \beta} & =A_{\alpha \beta}+\left(\zeta_{\alpha}^{c}+\tau_{\alpha}^{c}\right) \rho_{c d}\left(\zeta_{\beta}^{d}+\tau_{\beta}^{d}\right) \\
\left(g_{\tau, \sigma, \rho}\right)_{\alpha b} & =\left(\zeta_{\alpha}^{c}+\tau_{\alpha}^{c}\right) \rho_{c b} \\
\left(g_{\tau, \sigma, \rho}\right)_{a b} & =\rho_{a b}
\end{aligned}
$$

As may be seen from equation (2), $B_{\alpha \beta}$ represents the original horizontal kinetic energy metric. Noting that

$$
L_{\tau, \sigma, \rho}=\frac{1}{2} A_{\alpha \beta} \dot{x}^{\alpha} \dot{x}^{\beta}+\frac{1}{2} \rho_{a b}\left(\dot{\theta}^{a}+\left(\zeta_{\alpha}^{a}+\tau_{\alpha}^{a}\right) \dot{x}^{\alpha}\right)\left(\dot{\theta}^{b}+\left(\zeta_{\beta}^{b}+\tau_{\beta}^{b}\right) \dot{x}^{\beta}\right)-V^{\prime}\left(x^{\alpha}, \theta^{a}\right),
$$

one sees that $A_{\alpha \beta}$ plays an analogous role as the " $\tau$-horizontal" kinetic energy metric.

In the matching process, the terms $\sigma_{a b}, \rho_{a b}$, and $\tau_{\alpha}^{a}$ provide freedom to ensure that no inputs are required in uncontrolled directions. The artificial potential energy term $\tilde{V}\left(x^{\alpha}, \theta^{a}\right)$ provides more freedom. Still more freedom is introduced by allowing energy-conserving gyroscopic forces in the closed-loop system. After the requirements for matching are satisfied, the modified energy can be used to derive criteria for closed-loop stability. Any remaining freedom in the control parameters can then be used to satisfy these criteria and to tune controller performance.

The matching conditions are derived by comparing equations (8) and (10) and then choosing the control $u_{a}$ and the energy modification parameters $\tau, g_{\sigma}$, and $g_{\rho}$ so that (10) holds. In what follows, it will sometimes be convenient to use matrix notation, following conventions used by the robotics community. Define the local matrix forms of $g$ and $g_{\tau, \sigma, \rho}$ as follows,

$$
\boldsymbol{M}=\left(\begin{array}{ll}
{\left[g_{\alpha \beta}\right]} & {\left[g_{\alpha b}\right]} \\
{\left[g_{a \beta}\right]} & {\left[g_{a b}\right]}
\end{array}\right) \quad \text { and } \quad \boldsymbol{M}_{\tau, \sigma, \rho}=\left(\begin{array}{ll}
{\left[\left(g_{\tau, \sigma, \rho}\right)_{\alpha \beta}\right]} & {\left[\left(g_{\tau, \sigma, \rho}\right)_{\alpha b}\right]} \\
{\left[\left(g_{\tau, \sigma, \rho}\right)_{a \beta}\right]} & {\left[\left(g_{\tau, \sigma, \rho}\right)_{a b}\right]}
\end{array}\right)
$$

Define the concatenated state vector

$$
\boldsymbol{q}=\left[q^{k}\right]=\left(\begin{array}{l}
{\left[x^{\alpha}\right]} \\
{\left[\theta^{a}\right]}
\end{array}\right)
$$

Thus $\boldsymbol{q}$ is an $(r+n)$-vector where $r$ is the dimension of $\left[x^{\alpha}\right]$ and $n$ is the dimension of $\left[\theta^{a}\right]$. Next, define the matrix $C$ whose components are

$$
C_{i j}(\boldsymbol{q}, \dot{\boldsymbol{q}})=\sum_{k=1}^{r+n} \Gamma_{i j k} \dot{q}^{k}=\frac{1}{2} \sum_{k=1}^{r+n}\left(\frac{\partial M_{i j}}{\partial q^{k}}+\frac{\partial M_{i k}}{\partial q^{j}}-\frac{\partial M_{k j}}{\partial q^{i}}\right) \dot{q}^{k}
$$

where $M_{i j}$ is the $i j^{\text {th }}$ component of the mass matrix $\boldsymbol{M}$. The elements $\Gamma_{i j k}$ are the Christoffel symbols associated with $\boldsymbol{M}$. The matrix $\boldsymbol{C}$ is often called the "Coriolis and centripetal" matrix associated with $\boldsymbol{M}$. It can be shown that $\dot{M}-2 \boldsymbol{C}$ is skew-symmetric; see [24]. We make use of this property shortly when we develop sufficient conditions for matching with artificial gyroscopic forces. The matrix $\boldsymbol{C}_{\tau, \sigma, \rho}$ corresponding to $\boldsymbol{M}_{\tau, \sigma, \rho}$ is defined similarly to $\boldsymbol{C}$.

Let $\mathcal{E}$ represent the "Euler-Lagrange operator" defined as follows: given a Lagrangian $L$ and a generalized coordinate $y^{A}$,

$$
\mathcal{E}_{y^{A}}(L)=\frac{d}{d t} \frac{\partial L}{\partial \dot{y}^{A}}-\frac{\partial L}{\partial y^{A}}
$$


With these definitions, equations (8) may be written

$$
\left(\begin{array}{l}
{\left[\mathcal{E}_{x^{\alpha}}(L)\right]} \\
{\left[\mathcal{E}_{\theta^{a}}(L)\right]}
\end{array}\right)=\boldsymbol{M} \ddot{\boldsymbol{q}}+\boldsymbol{C} \dot{\boldsymbol{q}}+\left(\begin{array}{c}
{\left[V_{, \alpha}\right]} \\
{\left[V_{, a}\right]}
\end{array}\right)=\left(\begin{array}{c}
\mathbf{0} \\
{\left[u_{a}\right]}
\end{array}\right) .
$$

(By convention, commas in subscripts denote partial differentiation. For example, $V_{, a}$ is the partial derivative of $V$ with respect $\theta^{a}$.) Solving for $\ddot{\boldsymbol{q}}$ and substituting into the desired closed-loop equations (10) gives

$$
\left(\begin{array}{l}
{\left[\mathcal{E}_{x^{\alpha}}\left(L_{\tau, \sigma, \rho}\right)\right]} \\
{\left[\mathcal{E}_{\theta^{a}}\left(L_{\tau, \sigma, \rho}\right)\right]}
\end{array}\right)=\boldsymbol{M}_{\tau, \sigma, \rho} \boldsymbol{M}^{-1}\left\{-\boldsymbol{C} \dot{\boldsymbol{q}}+\left(\begin{array}{c}
{\left[-V_{, \alpha}\right]} \\
{\left[-V_{, a}+u_{a}\right]}
\end{array}\right)\right\}+\boldsymbol{C}_{\tau, \sigma, \rho} \dot{\boldsymbol{q}}+\left(\begin{array}{c}
{\left[V_{, \alpha}^{\prime}\right]} \\
{\left[V_{, a}^{\prime}\right]}
\end{array}\right)=\boldsymbol{\mathcal { S }} \dot{\boldsymbol{q}}
$$

where

$$
\mathcal{S}=-\mathcal{S}^{T}=\left(\begin{array}{ll}
{\left[\mathcal{S}_{\alpha \beta}\right]} & {\left[\mathcal{S}_{\alpha b}\right]} \\
{\left[\mathcal{S}_{a \beta}\right]} & {\left[\mathcal{S}_{a b}\right]}
\end{array}\right)
$$

To find the matching conditions, we partition the input into two components,

$$
u_{a}=u_{a}^{\mathrm{k} / \mathrm{g}}\left(x^{\alpha}, \theta^{a}, \dot{x}^{\alpha}, \dot{\theta}^{a}\right)+u_{a}^{\mathrm{p}}\left(x^{\alpha}, \theta^{a}\right),
$$

and match velocity-independent and velocity-dependent terms separately. The first term $u_{a}^{\mathrm{k} / \mathrm{g}}$ shapes the closed-loop kinetic energy and introduces gyroscopic forces into the closed-loop Euler-Lagrange equations. The second term $u_{a}^{\mathrm{p}}$ shapes the closed-loop potential energy.

Velocity-dependent terms. Referring to equations (17), we must find the velocity-dependent component of the control law $\left(u_{a}^{\mathrm{k} / \mathrm{g}}\right)$ and conditions on the system and control parameters such that

$$
\left.\boldsymbol{M}_{\tau, \sigma, \rho} \boldsymbol{M}^{-1}\left(\left(\begin{array}{c}
\mathbf{0} \\
{\left[u_{a}^{\mathrm{k} / \mathrm{g}}\right]}
\end{array}\right]\right)-\boldsymbol{C} \dot{\boldsymbol{q}}\right)+\boldsymbol{C}_{\tau, \sigma, \rho} \dot{\boldsymbol{q}}=\mathcal{S} \dot{\boldsymbol{q}} .
$$

Motivated by the form of the terms in (20), we let

$$
\left(\begin{array}{c}
\mathbf{0} \\
{\left[u_{a}^{\mathrm{k} / \mathrm{g}}\right]}
\end{array}\right)=\boldsymbol{U}\left(x^{\alpha}, \theta^{a}, \dot{x}^{\alpha}, \dot{\theta}^{a}\right) \dot{\boldsymbol{q}}
$$

where

$$
\boldsymbol{U}=\left(\begin{array}{cc}
\mathbf{0} & \mathbf{0} \\
{\left[U_{a \beta}\right]} & {\left[U_{a b}\right]}
\end{array}\right)
$$

Substituting in equation (20), we require

$$
\left[\boldsymbol{M}_{\tau, \sigma, \rho} \boldsymbol{M}^{-1}(\boldsymbol{U}-\boldsymbol{C})+\boldsymbol{C}_{\tau, \sigma, \rho}-\mathcal{S}\right] \dot{\boldsymbol{q}}=\mathbf{0}
$$

for all $\dot{\boldsymbol{q}} \in T_{q} Q$. Recognizing that $\boldsymbol{C}$ and $\boldsymbol{C}_{\tau, \sigma, \rho}$ are linear in velocity, these are $n$ equations which are quadratic in the components of velocity; these equations provide the necessary and sufficient conditions for matching as well as the functional form of the velocity-dependent control term $u_{a}^{\mathrm{k} / \mathrm{g}}$.

More modest conditions, which are sufficient for matching, are obtained by assuming that $\boldsymbol{U}$ and $\mathcal{S}$ are also linear in velocity and requiring that

$$
\boldsymbol{M}_{\tau, \sigma, \rho} \boldsymbol{M}^{-1}(\boldsymbol{U}-\boldsymbol{C})+\boldsymbol{C}_{\tau, \sigma, \rho}=\mathcal{S}
$$

By skew symmetry of $\mathcal{S}$, we need

$$
\left(\boldsymbol{M}_{\tau, \sigma, \rho} \boldsymbol{M}^{-1}(\boldsymbol{U}-\boldsymbol{C})+\boldsymbol{C}_{\tau, \sigma, \rho}\right)+\left(\left(\boldsymbol{U}^{T}-\boldsymbol{C}^{T}\right)\left(\boldsymbol{M}_{\tau, \sigma, \rho} \boldsymbol{M}^{-1}\right)^{T}+\boldsymbol{C}_{\tau, \sigma, \rho}^{T}\right)=\mathbf{0} .
$$


Recalling that $\dot{\boldsymbol{M}}_{\tau, \sigma, \rho}-2 \boldsymbol{C}_{\tau, \sigma, \rho}$ is skew symmetric, we may rewrite equation (23) as

$$
\boldsymbol{M}_{\tau, \sigma, \rho} \boldsymbol{M}^{-1}(\boldsymbol{U}-\boldsymbol{C})+\left(\boldsymbol{U}^{T}-\boldsymbol{C}^{T}\right)\left(\boldsymbol{M}_{\tau, \sigma, \rho} \boldsymbol{M}^{-1}\right)^{T}+\dot{\boldsymbol{M}}_{\tau, \sigma, \rho}=\mathbf{0} .
$$

For convenience, define

$$
\mathcal{M}=\left(\begin{array}{ll}
{\left[\mathcal{M}_{\alpha}^{\beta}\right.} \\
{\left[\mathcal{M}_{a}^{\beta}\right]} & {\left[\mathcal{M}_{\alpha}^{b}\right]} \\
{\left[\mathcal{M}_{a}^{b}\right]}
\end{array}\right)=\boldsymbol{M}_{\tau, \sigma, \rho} \boldsymbol{M}^{-1}
$$

Also, let tilde denote a component of a transposed matrix; for example, $\left[\tilde{\mathcal{M}}_{\alpha}^{b}\right]$ represents the upper right block of $\mathcal{M}^{T}$. Equation (24) implies that

$$
\begin{aligned}
& 0=\left(-\mathcal{M}_{\alpha}^{\gamma} C_{\gamma \beta}+\mathcal{M}_{\alpha}^{c}\left(U_{c \beta}-C_{c \beta}\right)\right)+\left(-\tilde{C}_{\alpha \gamma} \tilde{\mathcal{M}}_{\beta}^{\gamma}+\left(\tilde{U}_{\alpha c}-\tilde{C}_{\alpha c}\right) \tilde{\mathcal{M}}_{\beta}^{c}\right)+\left(\dot{\boldsymbol{M}}_{\tau, \sigma, \rho}\right)_{\alpha \beta} \\
& 0=\left(-\mathcal{M}_{\alpha}^{\gamma} C_{\gamma b}+\mathcal{M}_{\alpha}^{c}\left(U_{c b}-C_{c b}\right)\right)+\left(-\tilde{C}_{\alpha \gamma} \tilde{\mathcal{M}}_{b}^{\gamma}+\left(\tilde{U}_{\alpha c}-\tilde{C}_{\alpha c}\right) \tilde{\mathcal{M}}_{b}^{c}\right)+\left(\dot{\boldsymbol{M}}_{\tau, \sigma, \rho}\right)_{\alpha b} \\
& 0=\left(-\mathcal{M}_{a}^{\gamma} C_{\gamma \beta}+\mathcal{M}_{a}^{c}\left(U_{c \beta}-C_{c \beta}\right)\right)+\left(-\tilde{C}_{a \gamma} \tilde{\mathcal{M}}_{\beta}^{\gamma}+\left(\tilde{U}_{a c}-\tilde{C}_{a c}\right) \tilde{\mathcal{M}}_{\beta}^{c}\right)+\left(\dot{\boldsymbol{M}}_{\tau, \sigma, \rho}\right)_{a \beta} \\
& 0=\left(-\mathcal{M}_{a}^{\gamma} C_{\gamma b}+\mathcal{M}_{a}^{c}\left(U_{c b}-C_{c b}\right)\right)+\left(-\tilde{C}_{a \gamma} \tilde{\mathcal{M}}_{b}^{\gamma}+\left(\tilde{U}_{a c}-\tilde{C}_{a c}\right) \tilde{\mathcal{M}}_{b}^{c}\right)+\left(\dot{\boldsymbol{M}}_{\tau, \sigma, \rho}\right)_{a b}
\end{aligned}
$$

Solving equation (28) for $U_{a b}$, one finds that it is linear in velocity, since all other terms in (28) are linear in velocity. Substituting the solution for $U_{a b}$ into equation (26) or (27), one obtains the solution for $U_{a \beta}$, which is also linear in velocity. Substituting the control terms $U_{a b}$ and $U_{a \beta}$ into equation (25) gives the final conditions for matching. Since each term in (25) is linear in velocity, and the identity must hold for any velocity, there are $\frac{1}{2} r(r+1)$ independent equations which are linear in velocity. Since $\dot{x}^{\alpha}$ and $\dot{\theta}^{a}$ are independent, each of these $\frac{1}{2} r(r+1)$ equations can be decomposed into $r+n$ independent equations. In all, there are $\frac{1}{2} r(r+1)(r+n)$ partial differential equations. These equations involve the $n(n+r+1)$ unspecified functions $\tau_{\alpha}^{a}, \sigma_{a b}$, and $\rho_{a b}$, and their first partial derivatives. Matching involves using freedom available in $\tau_{\alpha}^{a}, \sigma_{a b}$, and $\rho_{a b}$, to solve these equations. Ideally, some parametric freedom will remain after the velocitydependent matching problem has been solved. If so, this freedom can be exploited in later analysis to help obtain conditions for closed-loop stability.

Note, in the procedure described above, that the components of $\mathcal{S}$ do not appear explicitly in the matching process; $\mathcal{S}$ is a product of the procedure, to be computed after matching has been achieved.

Velocity-independent terms. Since the terms in (17) involving the potential energy $V$ are independent of velocity, we require that

$$
\boldsymbol{M}_{\tau, \sigma, \rho} \boldsymbol{M}^{-1}\left(\begin{array}{c}
{\left[-V_{, \alpha}\right]} \\
{\left[-V_{, a}+u_{a}^{\mathrm{p}}\right]}
\end{array}\right)+\left(\begin{array}{c}
{\left[V_{, \alpha}^{\prime}\right]} \\
{\left[V_{, a}^{\prime}\right]}
\end{array}\right)=\left(\begin{array}{c}
\mathbf{0} \\
\mathbf{0}
\end{array}\right) .
$$

Written explicitly in terms of the component matrices

$$
\begin{aligned}
& M_{\tau, \sigma, \rho} M^{-1}
\end{aligned}
$$

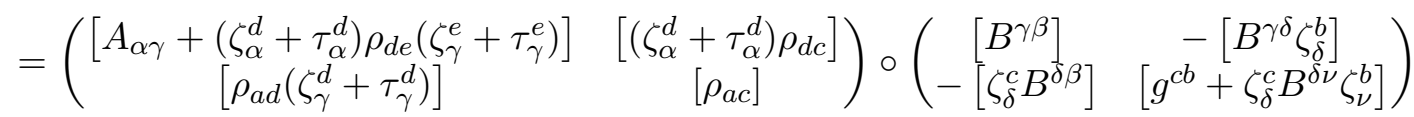

$$
\begin{aligned}
& =\left(\begin{array}{cc}
{\left[A_{\alpha \gamma} B^{\gamma \beta}+\left(\zeta_{\alpha}^{d}+\tau_{\alpha}^{d}\right) \rho_{d e} \tau_{\gamma}^{e} B^{\gamma \beta}\right]} & {\left[-A_{\alpha \gamma} B^{\gamma \delta} \zeta_{\delta}^{b}+\left(\zeta_{\alpha}^{c}+\tau_{\alpha}^{c}\right) \rho_{c d} D^{c b}\right]} \\
{\left[\rho_{a c} \tau_{\gamma}^{c} B^{\gamma \beta}\right]} & {\left[\rho_{a c} D^{c b}\right]}
\end{array}\right)
\end{aligned}
$$

where

$$
D^{a b}=g^{a b}-\tau_{\gamma}^{a} B^{\gamma \delta} \zeta_{\delta}^{b}
$$


Substituting (30) into (29), one obtains two equations. The second equation of (29) requires that

$$
0=\rho_{a c} \tau_{\gamma}^{c} B^{\gamma \beta}\left(-V_{, \beta}\right)+\rho_{a c} D^{c b}\left(u_{b}^{\mathrm{p}}-V_{, b}\right)+V_{, a}^{\prime} .
$$

Solving (32) for the control law gives

$$
u_{a}^{\mathrm{p}}=V_{, a}+D_{a c}\left(\tau_{\gamma}^{c} B^{\gamma \beta} V_{, \beta}-\rho^{c b} V_{, b}^{\prime}\right)
$$

The problem remains to find a condition such that the first equation in (29) holds under the control law (33). As shown in Appendix A, the appropriate "potential matching condition" is the following partial differential equation for $V^{\prime}\left(x^{\alpha}, \theta^{a}\right)$ :

$$
\begin{aligned}
0=\left(V_{, \alpha}^{\prime}-V_{, \alpha}\right) & -\left(\tau_{\alpha}^{c} \sigma_{c d} g^{d e}+\zeta_{\alpha}^{e}\right) D_{e f} \tau_{\gamma}^{f} B^{\gamma \beta} V_{, \beta} \\
& +\left(\left(\tau_{\alpha}^{c} \sigma_{c d} g^{d e}+\zeta_{\alpha}^{e}\right) D_{e f} \rho^{f b}-\tau_{\alpha}^{c} \sigma_{c d} \rho^{d b}-\zeta_{\alpha}^{b}-\tau_{\alpha}^{b}\right) V_{, b}^{\prime}
\end{aligned}
$$

Remark 3.1. If the potential energy remains unaltered by feedback $\left(V^{\prime}=V\right)$ and is independent of $\theta^{a}$, then condition (34) is satisfied by choosing

$$
\tau_{\alpha}^{a}=-\sigma^{a c} g_{c \alpha}
$$

If the potential energy is not modified in the uncontrolled directions $\left(V_{, \alpha}^{\prime}=V_{, \alpha}\right)$, then condition (34) is satisfied by choosing

$$
\tau_{\alpha}^{a}=-\sigma^{a c} g_{c \alpha} \quad \text { and } \quad \sigma^{a b}+\rho^{a b}=g^{a b} .
$$

This choice of $\tau_{\alpha}^{a}$ is common to several previous matching results, including the "first matching theorem" of [13], the "Euler-Poincaré matching conditions" of [14], and the "general matching conditions" stated in [12]. The choice of $\rho_{a b}$ is common to the "Euler-Poincaré matching conditions" and the "general matching conditions" of [12] where the problem of stabilizing the rotary inverted pendulum was considered. In all these previous cases, the choices were made to satisfy matching conditions for kinetic energy shaping rather than potential energy shaping. Because the term $\boldsymbol{M}_{\tau, \sigma, \rho} \boldsymbol{M}^{-1}$ appears in the kinetic shaping identity (20) and the potential shaping identity (29) in precisely the same way, it is not surprising that conditions developed for kinetic energy shaping also arise when shaping the potential energy.

\section{Dissipation}

To determine how dissipation, either natural or artificial, affects the closed-loop system (10), consider a more general version of the open-loop equations (8):

$$
\begin{aligned}
\frac{d}{d t} \frac{\partial L}{\partial \dot{x}^{\alpha}}-\frac{\partial L}{\partial x^{\alpha}} & =F_{\alpha} \\
\frac{d}{d t} \frac{\partial L}{\partial \dot{\theta}^{a}}-\frac{\partial L}{\partial \theta^{a}} & =u_{a}+F_{a} .
\end{aligned}
$$

The term $F_{\alpha}$ represents generalized forces which are inherent to the system, such as friction. The term $F_{a}$ represents some combination of natural forces within the system and user-defined control forces applied to it. With $u_{a}$ determined according to the procedure described in Section 3, we consider stability of the equilibrium (9), where it is assumed that $F_{\alpha}=F_{a}=0$ at the equilibrium. (See [18] for a general discussion of external forces in controlled Lagrangian and controlled Hamiltonian systems.) 
We assume that the control-modified energy $E_{\tau, \sigma, \rho}$ is a Lyapunov function in the conservative setting. That is, the energy and structure shaping control law $u_{a}=u_{a}^{\mathrm{k} / \mathrm{g}}+u_{a}^{\mathrm{p}}$ stabilizes the system about a minimum or a maximum of $E_{\tau, \sigma, \rho}$. Under this choice of feedback, the closed-loop dynamics with the external forces become

$$
\left(\begin{array}{l}
{\left[\mathcal{E}_{x^{\alpha}}\left(L_{\tau, \sigma, \rho}\right)\right]} \\
{\left[\mathcal{E}_{\theta^{a}}\left(L_{\tau, \sigma, \rho}\right)\right]}
\end{array}\right)=\mathcal{S} \dot{\boldsymbol{q}}+\boldsymbol{M}_{\tau, \sigma, \rho} \boldsymbol{M}^{-1}\left(\begin{array}{l}
{\left[F_{\alpha}\right]} \\
{\left[F_{a}\right]}
\end{array}\right)
$$

Referring to equation (30) for $\boldsymbol{M}_{\tau, \sigma, \rho} \boldsymbol{M}^{-1}$, the control-modified energy satisfies

$$
\begin{aligned}
\frac{d}{d t} E_{\tau, \sigma, \rho} & =\dot{\boldsymbol{q}}^{T} \boldsymbol{M}_{\tau, \sigma, \rho} \boldsymbol{M}^{-1}\left(\begin{array}{c}
{\left[F_{\alpha}\right]} \\
{\left[F_{a}\right]}
\end{array}\right) \\
& =\dot{x}^{\alpha} A_{\alpha \gamma} B^{\gamma \beta}\left(F_{\beta}-\zeta_{\beta}^{b} F_{b}\right)+\left(\dot{\theta}^{a}+\dot{x}^{\alpha}\left(\zeta_{\alpha}^{a}+\tau_{\alpha}^{a}\right)\right) \rho_{a c}\left(D^{c b} F_{b}+\tau_{\gamma}^{c} B^{\gamma \beta} F_{\beta}\right) .
\end{aligned}
$$

The desired equilibrium will remain stable in the presence of damping if $\dot{E}_{\tau, \sigma, \rho}$ is semidefinite with opposite sign to $E_{\tau, \sigma, \rho}$.

Remark 4.1. Feedback dissipation with no physical damping. Suppose that $F_{\alpha}=0$ and that $F_{a}$ can be specified as a dissipative feedback control law. Then

$$
\frac{d}{d t} E_{\tau, \sigma, \rho}=\left(D^{a b} \rho_{b c}\left(\dot{\theta}^{c}+\left(\zeta_{\alpha}^{c}+\tau_{\alpha}^{c}\right) \dot{x}^{\alpha}\right)-\zeta_{\alpha}^{a} B^{\alpha \beta} A_{\beta \gamma} \dot{x}^{\gamma}\right) F_{a}
$$

Therefore, choosing

$$
F_{a}=u_{a}^{\mathrm{diss}}=k_{a b}^{\mathrm{diss}}\left(D^{b c} \rho_{c d}\left(\dot{\theta}^{d}+\left(\zeta_{\alpha}^{d}+\tau_{\alpha}^{d}\right) \dot{x}^{\alpha}\right)-\zeta_{\alpha}^{d} B^{\alpha \beta} A_{\beta \gamma} \dot{x}^{\gamma}\right)
$$

makes $\dot{E}_{\tau, \sigma, \rho}$ quadratic. The modified energy rate can clearly be made either positive or negative semidefinite, as desired, by choosing $k_{a b}^{\text {diss }}$ appropriately. For example, if $E_{\tau, \sigma, \rho}$ is positive definite at the desired equilibrium, one may choose $k_{a b}^{\text {diss }}$ negative definite so that $\dot{E}_{\tau, \sigma, \rho}$ will be negative semidefinite. The equilibrium thus remains stable and asymptotic stability may be assessed using LaSalle's invariance principle.

When the system is subject to physical damping, asymptotic stabilization is more subtle. By "physical damping," we mean dissipation which opposes velocity in the sense that

$$
\dot{x}^{\alpha} F_{\alpha}+\dot{\theta}^{a} F_{a}<0
$$

whenever the velocity is nonzero. Consider the simplest case of linear damping

$$
\left(\begin{array}{l}
{\left[F_{\alpha}\right]} \\
{\left[F_{a}\right]}
\end{array}\right)=-\boldsymbol{R} \dot{\boldsymbol{q}}
$$

where $\boldsymbol{R}$ is a matrix of damping constants. Then

$$
\begin{aligned}
\frac{d}{d t} E_{\tau, \sigma, \rho} & =-\dot{\boldsymbol{q}}^{T} \boldsymbol{M}_{\tau, \sigma, \rho} \boldsymbol{M}^{-1} \boldsymbol{R} \dot{\boldsymbol{q}} \\
& =-\frac{1}{2} \dot{\boldsymbol{q}}^{T}\left(\boldsymbol{M}_{\tau, \sigma, \rho} \boldsymbol{M}^{-1} \boldsymbol{R}+\boldsymbol{R}^{T} \boldsymbol{M}^{-1} \boldsymbol{M}_{\tau, \sigma, \rho}\right) \dot{\boldsymbol{q}}
\end{aligned}
$$

If $\boldsymbol{M}_{\tau, \sigma, \rho}, \boldsymbol{M}$, and the symmetric part of $\boldsymbol{R}$ are all positive definite, one might expect that

$$
\boldsymbol{M}_{\tau, \sigma, \rho} \boldsymbol{M}^{-1} \boldsymbol{R}+\boldsymbol{R}^{T} \boldsymbol{M}^{-1} \boldsymbol{M}_{\tau, \sigma, \rho}>0
$$


and therefore that $\dot{E}_{\tau, \sigma, \rho} \leq 0$. In general, this is not the case. Thus, one must take special care when considering the effect of linear damping in systems controlled using kinetic energy shaping feedback. The example described in Section 5 illustrates this issue. The importance of physical damping in general controlled Lagrangian systems is acknowledged in [3]. The issue is also discussed, for special classes of controlled Lagrangian systems, in [29] and [30].

The problem of physical dissipation is not unique to the method of controlled Lagrangians, but arises whenever the kinetic energy metric is modified through feedback. As observed by a reviewer, and also by Gómez-Estern \& van der Schaft [19], the problem can be understood as a lack of passivity in the closed-loop system. Taking $E_{\tau, \sigma, \rho}$ as the natural storage function, and referring to (36), one sees that the passive output for the closed-loop system is $\boldsymbol{M}^{-1} \boldsymbol{M}_{\tau, \sigma, \rho} \dot{\boldsymbol{q}}$. The dissipative forces (39) depend on the open-loop passive output $\dot{\boldsymbol{q}}$ rather than the closed-loop passive output. Observing that $\dot{E}_{\tau, \sigma, \rho}$ fails to be negative semidefinite under these damping forces is equivalent to observing that the theorem of passivity does not apply.

\section{Example: The Pendulum on a Cart}

To illustrate the results of Sections 3 and 4, and to maintain continuity with previous work, we consider the problem of stabilizing an inverted pendulum on a cart. In [13] the "simplified matching conditions" led to a choice of feedback which recovered a closed-loop Lagrangian system with no gyroscopic forces. Conservation of the modified total energy and the modified translational momentum allowed the construction of a control Lyapunov function for the desired equilibrium. There, the desired equilibrium was the pendulum in the upright position with the cart moving at a prescribed constant velocity. Conservation of the translational momentum was used to reduce the order of the dynamics, and the desired equilibrium was shown to be a maximum of the closed-loop potential energy and of the horizontal component of the closed-loop kinetic energy.

In [6], the approach was made more powerful by introducing an additional control term to modify the closedloop potential energy. The modified potential energy broke the symmetry, and the translational momentum was no longer conserved. In this case, the desired equilibrium, corresponding to the pendulum in the upright position and the cart at rest at the origin, was made into a maximum of the closed-loop energy.

Here, we go a step further by allowing gyroscopic forces in the closed-loop system. The desired equilibrium, corresponding to the pendulum in the upright position and the cart at rest at the origin, becomes a minimum of the control-modified total energy. The basin of stability is the set of states for which the pendulum is elevated above the horizontal line through its pivot.

This same example has been considered in numerous other papers on energy shaping, including [1], [2], and [20]. Two features of our control design and analysis distinguish our treatment from others. First, the closed-loop Lagrangian system includes artificial gyroscopic forces, illustrating that such energy-conserving forces can play a useful role in the matching process. Second, we consider the issue of physical damping and its effect on the closed-loop dynamics. As the example illustrates, such analysis is crucial if one wishes to implement a feedback control law which modifies a system's kinetic energy.

\subsection{Conservative Model}

The inverted pendulum on a cart is depicted in Figure 3. The cart location is denoted by $s$ and the pendulum angle by $\phi$. A control force $u$ is applied to the cart. The configuration space is $Q=\mathbb{R} \times S^{1}$ and the uncontrolled dynamics are invariant under the action of $G=S^{1}$ on $Q$. (See $[6,13]$.) 
Figure 3: Pendulum on a cart.

We introduce the following nondimensional variables,

$$
\bar{s}=\frac{s}{l}, \quad \gamma=\frac{M+m}{m}, \quad \text { and } \quad T=\omega t \quad \text { where } \quad \omega=\sqrt{\frac{g}{l}} .
$$

We denote differentiation with respect to nondimensional time $T$ with an overdot. Using the notation of Bloch, Leonard, \& Marsden [13], and dropping the overbar from $\bar{s}$, the nondimensional Lagrangian is

$$
L=\frac{1}{2}\left(\begin{array}{c}
\dot{\phi} \\
\dot{s}
\end{array}\right)^{T}\left(\begin{array}{cc}
1 & \cos \phi \\
\cos \phi & \gamma
\end{array}\right)\left(\begin{array}{c}
\dot{\phi} \\
\dot{s}
\end{array}\right)-\cos \phi
$$

In the conservative setting, the open-loop equations of motion for this system are

$$
\left(\begin{array}{l}
\mathcal{E}_{\phi}(L) \\
\mathcal{E}_{s}(L)
\end{array}\right)=\left(\begin{array}{l}
0 \\
u
\end{array}\right)
$$

Because this is a two-degree-of-freedom problem, we may define the scalar parameters

$$
\tau=\left[\tau_{\alpha}^{a}\right], \quad \sigma=\left[\sigma_{a b}\right], \quad \text { and } \quad \rho=\left[\rho_{a b}\right]
$$

without ambiguity. From (14), the modified kinetic energy metric is

$$
\boldsymbol{M}_{\tau, \sigma, \rho}=\left(\begin{array}{cc}
\left(1-\frac{1}{\gamma} \cos ^{2} \phi\right)+\sigma \tau^{2}+\rho\left(\tau+\frac{1}{\gamma} \cos \phi\right)^{2} & \rho\left(\tau+\frac{1}{\gamma} \cos \phi\right) \\
\rho\left(\tau+\frac{1}{\gamma} \cos \phi\right) & \rho
\end{array}\right)
$$

As described at the end of Section 3, we first compute the terms $U_{a b}$ and $U_{a \beta}$ from (27) and (28). We then consider the two PDEs obtained from (25). To preserve symmetry in the modified kinetic energy, $\tau, \sigma$, and $\rho$ are independent of the cart position $s$. The two PDEs reduce to a single ODE in the three unknown functions $\tau, \sigma$, and $\rho$. As a two degree of freedom, single-input system, the cart-pendulum problem is obviously quite special. In general, solving the $\frac{1}{2} r(r+1)(r+n)$ PDEs for matching may be challenging.

Aided by Mathematica, one finds that the following choices satisfy the equation:

$$
\begin{aligned}
\tau & =\frac{2}{\cos \phi}, \\
\sigma & =\frac{4-\left(2+\cos ^{3} \phi\right)\left(1-\frac{1}{\gamma} \cos ^{2} \phi\right)}{4 \cos \phi}, \text { and } \\
\rho & =\frac{2}{\cos \phi\left(1-\frac{1}{\gamma} \cos ^{2} \phi\right)} .
\end{aligned}
$$


For this matching solution, no parametric freedom remains in the control-modified kinetic energy. Remarkably, the closed-loop kinetic energy metric is positive definite and the parametric freedom obtained through potential shaping is sufficient to allow stabilization.

The velocity-dependent component of the energy shaping control law is

$$
\begin{gathered}
u^{\mathrm{k} / \mathrm{g}}=\frac{1}{2\left(\gamma+\cos ^{2} \phi\right)^{2}}\left(\left(\gamma^{2}\left(5 \gamma-4 \cos ^{2} \phi\right) \sec \phi \tan \phi-3\left(5 \gamma+2 \cos ^{2} \phi\right) \sin \phi \cos ^{2} \phi\right) \dot{\phi}^{2}\right. \\
\left.+\left(\gamma^{2}\left(\gamma-2 \cos ^{2} \phi\right) \tan \phi-3 \gamma \sin \phi \cos ^{3} \phi\right) \dot{\phi} \dot{s}\right)
\end{gathered}
$$

Next, solving the potential shaping PDE (34) and substituting the solution into (33), we find that we may choose

$$
u^{\mathrm{p}}=\frac{1}{2\left(\gamma+\cos ^{2} \phi\right)}\left(4 \gamma^{2} \tan \phi+\cos \phi\left(\gamma-\cos ^{2} \phi\right)^{2} \frac{d v(\varphi(\phi, s))}{d \varphi}\right),
$$

where $v(\cdot)$ is an arbitrary $C^{1}$ function and where

$$
\varphi(\phi, s)=s+6 \operatorname{arctanh}\left(\tan \left(\frac{\phi}{2}\right)\right) .
$$

Note that $\varphi$ is well-defined for all $s$ and for all $\phi \in\left(-\frac{\pi}{2}, \frac{\pi}{2}\right)$. The input $u^{\mathrm{p}}$ effectively alters the system's potential energy; the modified potential function is

$$
V^{\prime}(\phi, s)=\left(\left(\frac{1}{\cos ^{2} \phi}-1\right)+v(\varphi(\phi, s))\right) .
$$

Figure (4) shows level sets of the modified potential energy with $v(\varphi)=\frac{1}{2} \kappa \varphi^{2}$ and with $\kappa=0.5$.

Figure 4: Level sets of $V^{\prime}(\phi, s)$.

\section{Letting}

$$
u=u^{\mathrm{k} / \mathrm{g}}+u^{\mathrm{p}},
$$

the closed-loop equations of motion are

$$
\left(\begin{array}{l}
\mathcal{E}_{\phi}\left(L_{\tau, \sigma, \rho}\right) \\
\mathcal{E}_{s}\left(L_{\tau, \sigma, \rho}\right)
\end{array}\right)=\left(\begin{array}{cc}
0 & \varsigma \\
-\varsigma & 0
\end{array}\right)\left(\begin{array}{l}
\dot{\phi} \\
\dot{s}
\end{array}\right)
$$


where the control-modified Lagrangian is

$$
L_{\tau, \sigma, \rho}=\frac{1}{2}\left(\begin{array}{c}
\dot{\phi} \\
\dot{s}
\end{array}\right)^{T} M_{\tau, \sigma, \rho}\left(\begin{array}{c}
\dot{\phi} \\
\dot{s}
\end{array}\right)-V^{\prime}(\phi, s)
$$

and where

$$
\varsigma=-\frac{\gamma}{\left(\gamma-\cos ^{2} \phi\right)^{2}}\left(\left(\gamma-3 \cos ^{2} \phi\right) \sec ^{2} \phi \tan \phi(3 \dot{\phi}+\dot{s} \cos \phi)\right) .
$$

Proposition 5.1. The control law (45), with $u^{\mathrm{k} / \mathrm{g}}$ given by (43) and $u^{\mathrm{p}}$ given by (44), and with

$$
v(\varphi)=\frac{1}{2} \kappa \varphi^{2},
$$

stabilizes the equilibrium at the origin provided $\kappa>0$. Moreover, for all initial states in the set

$$
W=\left\{(\phi, s, \dot{\phi}, \dot{s}) \in S^{1} \times \mathbb{R}^{3}|| \phi \mid<\frac{\pi}{2}\right\},
$$

trajectories exist for all time and are confined to compact, invariant, level sets of the modified total energy

$$
E_{\tau, \sigma, \rho}=\frac{1}{2}\left(\begin{array}{c}
\dot{\phi} \\
\dot{s}
\end{array}\right)^{T} \boldsymbol{M}_{\tau, \sigma, \rho}\left(\begin{array}{c}
\dot{\phi} \\
\dot{s}
\end{array}\right)+\left(\left(\frac{1}{\cos ^{2} \phi}-1\right)+v(\varphi(\phi, s))\right) .
$$

Proof: The desired equilibrium is a strict minimum of the control-modified energy, which is conserved under the closed-loop dynamics. Thus $E_{\tau, \sigma, \rho}$ is a Lyapunov function and stability of the origin follows by Lyapunov's second method. Level sets of $E_{\tau, \sigma, \rho}$ which are contained in $W$ are invariant because $E_{\tau, \sigma, \rho}$ is conserved. To check that these level sets are compact, apply the change of coordinates

$$
(\phi, s, \dot{\phi}, \dot{s}) \mapsto(\chi, s, \dot{\chi}, \dot{s})
$$

where $\chi=\tan \phi$. This change of variables defines a diffeomorphism from $W$ to $\mathbb{R}^{4}$. In the transformed coordinates, the control-modified energy $E_{\tau, \sigma, \rho}$ is radially unbounded. It follows that the level sets of $E_{\tau, \sigma, \rho}$ contained in $W$ are compact; see [21].

\subsection{Conservative Model with Feedback Dissipation}

Next, we apply dissipative feedback, as described in Remark 4.1. The complete feedback control law is

$$
u=u^{\mathrm{k} / \mathrm{g}}+u^{\mathrm{p}}+u^{\mathrm{diss}} .
$$

Setting

$$
u^{\mathrm{diss}}=k_{\mathrm{diss}}\left(-\frac{2 \sec ^{2} \phi\left(\gamma+\cos ^{2} \phi\right)(3 \dot{\phi}+\dot{s} \cos \phi)}{\left(\gamma-\cos ^{2} \phi\right)^{2}}\right),
$$

where $k_{\text {diss }}$ is a dimensionless dissipative control gain, gives

$$
\dot{E}_{\tau, \sigma, \rho}=k_{\text {diss }}\left(\frac{2 \sec ^{2} \phi\left(\gamma+\cos ^{2} \phi\right)(3 \dot{\phi}+\dot{s} \cos \phi)}{\left(\gamma-\cos ^{2} \phi\right)^{2}}\right)^{2} .
$$

Choosing $k_{\text {diss }}<0$ makes $\dot{E}_{\tau, \sigma, \rho}$ negative semidefinite.

Proposition 5.2. The control law (50), with $u^{\mathrm{k} / \mathrm{g}}$ and $u^{\mathrm{p}}$ given as in Proposition 5.1 and with $u^{\mathrm{diss}}$ given by (51), asymptotically stabilizes the origin provided $k_{\mathrm{diss}}<0$. The region of attraction is the set $W$ (48) of states for which the pendulum is elevated above the horizontal plane.

Proof: The proof is given in Appendix B. 


\subsection{Dissipative Model}

We have shown that one may choose $\kappa$ to make the equilibrium a strict minimum of the control-modified energy $E_{\tau, \sigma, \rho}$. We have also shown that one may choose $k_{\text {diss }}$ to drive $E_{\tau, \sigma, \rho}$ to that minimum value, in the absence of other dissipative forces. Interestingly, when $k_{\text {diss }}=0$ and physical damping is present, the control law does not provide asymptotic stability. Suppose a damping force opposes the cart's motion in proportion to the cart's velocity. Suppose also that a damping moment opposes the pendulum's motion in proportion to the pendulum angular rate. This simple linear damping destabilizes the inverted equilibrium unless it is properly countered through feedback. This phenomenon is consistent with previous control designs based on the method of controlled Lagrangians, as discussed in [29].

Assume that the closed-loop system described in Section 5.1 is subject to (nondimensional) dissipative external forces of the form

$$
\left[F_{\alpha}\right]=-d_{\phi} \dot{\phi} \quad \text { and } \quad\left[F_{a}\right]=-d_{s} \dot{s}
$$

where $d_{\phi}$ and $d_{s}$ are dimensionless damping constants. We assume that $d_{\phi}>0$. The value of $d_{s}$, on the other hand, can be modified directly through feedback. Indeed, one may impose arbitrary dissipative forces in the controlled directions and may thus modify the components in the bottom row of the matrix $\boldsymbol{R}$ through feedback. Regardless, it can be shown that there is no choice of linear feedback dissipation which makes inequality (40) hold for this example. (The proof is an extension of the proof of Lemma 4.2 in [26].) Because $\dot{E}_{\tau, \sigma, \rho}$ cannot be made negative semidefinite when $d_{\phi}>0, E_{\tau, \sigma, \rho}$ is no longer a Lyapunov function in this case.

Rather than search for a new Lyapunov function, we analyze nonlinear stability locally using Lyapunov's first method. That is, we examine the spectrum associated with the linearized dynamics. The local analysis provides a valuable assessment of the nonlinear controller's performance. In practice, one would not likely implement a nonlinear control law whose local performance compares poorly with available linear control laws.

As will be shown, the given controller, with an appropriate choice of linear feedback dissipation, is still quite effective at stabilizing the desired equilibrium. Moreover, even though we lose the Lyapunov function, and thus the proof of a large region of attraction, simulations suggest that the region of attraction remains quite large.

Encouraged by converse theorems for Lyapunov stability, one might search for a new Lyapunov function for the given controller. One approach might involve complementing $\dot{E}_{\tau, \sigma, \rho}$ with another function, and an appropriately defined switching sequence, such that the pair of functions proves asymptotic stability; see [15], for example. A more challenging approach, perhaps, would be to seek a new modified energy for the given control law, for which the equilibrium is a minimum (or a maximum) and for which a dissipation inequality such as (40) can be made to hold with suitably defined feedback dissipation. (In the case of nonlinear damping, the inequality (40) would have to be modified. Nonlinear damping often arises in real systems, including the system considered here; see [26].) Additional freedom can be obtained in the matching process by relaxing the symmetry requirements and allowing a more general Ehresmann connection; see [5].

Linearizing the closed-loop equations about the desired equilibrium gives

$$
\dot{\boldsymbol{q}}=\mathbf{A} \boldsymbol{q}+\text { h.o.t., }
$$

where $\boldsymbol{q}=\left[\begin{array}{llll}\phi & s & \dot{\phi} & \dot{s}\end{array}\right]^{T}$ is the state vector and the state matrix is

$$
\mathbf{A}=\left(\begin{array}{cccc}
0 & 0 & 1 & 0 \\
0 & 0 & 0 & 1 \\
-a-3 b \kappa & -b \kappa & -\frac{d_{\phi} \gamma}{\gamma-1}+3 c k_{\mathrm{diss}} & \frac{d_{s}}{\gamma-1}+c k_{\mathrm{diss}} \\
2 a+3 b \kappa+\frac{1}{\gamma+1} & b \kappa & -\frac{d_{\phi}}{\gamma-1}-3 c k_{\mathrm{diss}} & -\frac{d_{s}}{\gamma-1}-c k_{\mathrm{diss}}
\end{array}\right)
$$


with

$$
a=\frac{\gamma}{\gamma+1}, \quad b=\frac{\gamma-1}{2(\gamma+1)}, \quad \text { and } \quad c=\frac{2(\gamma+1)}{(\gamma-1)^{3}} .
$$

We point out that $\kappa$ and $k_{\text {diss }}$ are control parameters and $\gamma$ and $d_{\phi}$ are system parameters. Because the dissipative force corresponding to the parameter $d_{s}$ enters in the controlled direction, this parameter can be modified through feedback; still, we treat $d_{s}$ as a system parameter. Because $\gamma>1$, the constants $a, b$, and $c$ are all positive. We examine stability in the $\kappa$ - $k_{\text {diss }}$ parameter space for different system parameter values. Specifically, using Routh's criterion, we find values of $\kappa$ and $k_{\text {diss }}$ such that every eigenvalue of $\mathbf{A}$ has a negative real part for given system parameter values. We use Routh's criterion to find conditions on $\kappa$ and $k_{\text {diss }}$ such that exponential stability is guaranteed.

The eigenvalues $\lambda$ of $\mathbf{A}$ satisfy the fourth order polynomial

$$
\lambda^{4}+p_{3} \lambda^{3}+p_{2} \lambda^{2}+p_{1} \lambda+p_{0}=0
$$

where the coefficients are

$$
\begin{array}{ll}
p_{0}=b \kappa & p_{2}=a+\frac{d_{\phi} d_{s}}{\gamma-1}+2 b \kappa+c d_{\phi} k_{\text {diss }} \\
p_{1}=\frac{-d_{s}}{\gamma-1}-b d_{\phi} \kappa-c k_{\text {diss }} & p_{3}=\frac{\gamma d_{\phi}+d_{s}}{\gamma-1}-2 c k_{\text {diss }}
\end{array}
$$

Necessary and sufficient conditions for every eigenvalue to have negative real part are that

$$
p_{0}>0, \quad p_{1}>0, \quad p_{2}>0, \text { and } p_{3}>0,
$$

and that

$$
\begin{aligned}
\delta & =p_{2} p_{3}-p_{1} \quad>0 \\
\delta_{1} & =\delta p_{1}-p_{3}^{2} p_{0}>0 .
\end{aligned}
$$

Figure 5: Stabilizing values of control parameters $\left(d_{\phi}=d_{s}=0\right)$ in gray shaded area.

First, consider the simpler case where there is no physical dissipation, i.e., $d_{\phi}=d_{s}=0$. Figure 5 shows the curves

$$
p_{0}=0, \quad p_{1}=0, \quad p_{2}=0, \quad p_{3}=0, \quad \delta=0, \quad \delta_{1}=0
$$


in the $\kappa-k_{\text {diss }}$ plane. The hashed areas are control parameter values for which the conditions for asymptotic stability are violated. The region of stabilizing control parameter values is seen to be the entire fourth quadrant, which is consistent with Remark 4.1.

If $d_{s}$ and $d_{\phi}$ are nonzero, the region of stabilizing control parameter values shown in Figure 5 changes slightly.

Proposition 5.3. If $\sqrt{2}>d_{\phi} \geq 0$ and $d_{s}>-\gamma d_{\phi}$, then there exist control parameter values $\kappa$ and $k_{\mathrm{diss}}$ which exponentially stabilize the origin of the linearized dynamics (54)-(55).

\section{Proof: See Appendix C.}

The proof of the above result also shows that if $k_{\text {diss }}=0$, one can not find a stabilizing value of $\kappa$ when $d_{s}>0$ and $d_{\phi}>0$. That is, the control law developed for the conservative system model will fail to stabilize a system with generic, linear damping. One must introduce feedback dissipation to stabilize the system.

As an alternative to the feedback dissipation term (51), one may instead apply feedback which makes $d_{s}$ negative. (Although we have treated $d_{s}$ as a system parameter, the damping force associated with this parameter occurs in the controlled direction, so one may effectively change $d_{s}$ through feedback.) If $d_{s}$ satisfies $-\gamma d_{\phi}<d_{s}<0$, one may obtain closed-loop stability even with $k_{\text {diss }}=0$; see the sketch on the right-hand side of Figure 6. Applying feedback to make $d_{s}$ negative corresponds to reversing the natural damping force acting on the cart.

Proposition 5.3 asserts that, under quite reasonable conditions on the physical parameter values, there exist control parameter values which exponentially stabilize the linearized dynamics. For these parameter values, it follows from Lyapunov's first method that the nonlinear system is locally exponentially stable. In fact, simulations suggest that the region of attraction is once again the set $W$ of states for which the pendulum is elevated above the horizontal plane. We point out that exponential stability of the linearized dynamics also implies that the control law is, at least locally, robust to small uncertainties in the model parameters.

Figure 6: Stabilizing control parameter values (in gray) for $\gamma=2$ and $d_{\phi}=0.1$ with (a) $d_{s}=0.05$ and (b) $d_{s}=-0.05$. The solid line is $\delta_{1}=0$; the dashed line is $\delta=0$.

Numerical results. Figure 6 shows the region of stabilizing control parameter values for particular values of the system parameters. We fix $\gamma=2$ and $d_{\phi}=0.1$ and consider the two cases $d_{s}=0.05$ and $d_{s}=-0.05$. Figure 6 (a) shows that, when $d_{s}=0.05$, the $\kappa$-axis lies outside the region of stabilizing control parameter values. Thus, in absence of feedback dissipation (i.e., with $k_{\text {diss }}=0$ ), no value of the control parameter $\kappa$ 
can stabilize the system when $d_{s}>0$. When $d_{s}<0$, however, a portion of the $\kappa$-axis lies within the region of stabilizing control parameter values. This observation is illustrated in Figure $6(\mathrm{~b})$ for the case $d_{s}=-0.05$.

Figure 7: Pendulum angle and cart location versus time with $d_{\phi}=0$ (solid) and $d_{\phi}=0.1$ (dashed).

Figure 8: Control-modified energy $E_{\tau, \sigma, \rho}$ versus time with $d_{\phi}=0$ (solid) and $d_{\phi}=0.1$ (dashed). Note the non-monotonic convergence in the case where physical damping is present.

¿From Figure $6(\mathrm{~b})$, we see that the values $\kappa=0.5$ and $k_{\text {diss }}=-0.25$ lie in the region of stabilizing parameter values. Figure 7 shows closed-loop system trajectories with these parameter choices in response to the (nondimensional) initial conditions

$$
\phi(0)=\left(\frac{89}{90}\right) \frac{\pi}{2} \quad s(0)=0 \quad \dot{\phi}(0)=\pi \quad \dot{s}(0)=0 .
$$

Note that this initial state is far outside the region where the linearized equations provide a reasonable model for the nonlinear dynamics. It corresponds to an almost horizontal pendulum which is rotating downward. We assume a perfect actuator; the magnitude of the control force is unlimited and the actuator responds instantaneously to reference commands.

Figure 7 shows two cases. In the first case, denoted by solid lines, only feedback dissipation is applied $\left(d_{\phi}=d_{s}=0\right)$. In the second case, denoted by dashed lines, there is also physical damping in the unactuated 
direction $\left(d_{\phi}=0.1\right)$ and "reversed damping" in the actuated direction $\left(d_{s}=-0.05\right)$. In both cases, the system converges to the desired equilibrium, although more slowly in the latter case.

Figure 8 shows the control modified energy for the two cases described above. In both cases, the energy decays to its minimum value. In the presence of physical damping, however, the function does not decay monotonically. The control-modified total energy is not a Lyapunov function when physical damping is present.

\section{Conclusions}

We have modified the method of controlled Lagrangians, as presented in [13] and [6], to include artificial gyroscopic forces in the closed-loop system. These forces conserve the feedback-modified system energy and thus preserve its role as a control Lyapunov function. The introduction of gyroscopic forces allows additional freedom which can be used to expand the basin of stability and to tune the closed-loop system performance. Having stabilized a given system in this way, one may introduce feedback dissipation in an effort to achieve asymptotic stability. While the approach described here is less general than the formulation given in [18], it is more algorithmic.

The control design technique was applied to the problem of stabilizing an inverted pendulum on a cart. The control-modified energy was constructed such that the desired equilibrium is a minimum. The addition of feedback dissipation provides asymptotic stability within a region of attraction that contains all states for which the pendulum is elevated above the horizontal plane. Despite these results, generic physical damping makes the control-modified energy rate indefinite, thus invalidating the nonlinear stability argument. However, in this case, linear stability analysis provides a range of system and control parameter values which ensure that the desired equilibrium is locally exponentially stable. Moreover, simulations suggest that the region of attraction remains quite large.

As is clearly demonstrated by the example described here, physical damping must be carefully considered for systems whose kinetic energy has been modified through feedback. In previous work, such as [29], the stabilization problem was broken into three steps. The first step involved stabilizing a conservative model of the system. Parameters for the control-modified kinetic energy metric were chosen in this step. The second step involved adding feedback dissipation to the conservative system model. The third step involved considering physical damping and, if necessary, modifying the system damping through feedback. It may be advantageous to instead consider physical damping at the outset, that is, to choose parameters for the control-modified kinetic energy metric and for feedback dissipation at the same time.

The example of a pendulum on a cart motivates further study. For example, encouraged by the local analysis and simulation results, one might take a more general approach to energy shaping to search for a true Lyapunov function for the given closed-loop system. Alternatively, one might consider the problem of proving (non-monotonic) convergence of the control-modified energy in cases where the rate of change is indefinite. Of the two possible approaches, the former is more consistent with the control design philosophy and is perhaps more promising. 


\section{A The Potential Energy Matching Condition}

The upper term of equation (29) requires that

$$
0=\left(A_{\alpha \gamma}+\left(\zeta_{\alpha}^{d}+\tau_{\alpha}^{d}\right) \rho_{d e} \tau_{\gamma}^{e}\right) B^{\gamma \beta}\left(-V_{, \beta}\right)-\left(A_{\alpha \gamma} B^{\gamma \delta} \zeta_{\delta}^{b}-\left(\zeta_{\alpha}^{c}+\tau_{\alpha}^{c}\right) \rho_{c d} D^{d b}\right)\left(u_{b}^{\mathrm{p}}-V_{, b}\right)+V_{, \alpha}^{\prime} .
$$

Noting that

$$
A_{\alpha \gamma} B^{\gamma \beta}=\delta_{\alpha}^{\beta}+\tau_{\alpha}^{c} \sigma_{c d} \tau_{\gamma}^{d} B^{\gamma \beta}
$$

from the definition (13) of $A_{\alpha \beta}$, we may rewrite (60) as

$$
\begin{aligned}
0=\left(-V_{, \alpha}\right)+ & \left(\tau_{\alpha}^{c} \sigma_{c d} \rho^{d e}+\left(\zeta_{\alpha}^{e}+\tau_{\alpha}^{e}\right)\right)\left(\rho_{e f} \tau_{\gamma}^{f} B^{\gamma \beta}\right)\left(-V_{, \beta}\right) \\
& -\left(A_{\alpha \gamma} B^{\gamma \delta} \zeta_{\delta}^{b}-\left(\zeta_{\alpha}^{c}+\tau_{\alpha}^{c}\right) \rho_{c d} D^{d b}\right)\left(u_{b}^{\mathrm{p}}-V_{, b}\right)+V_{, \alpha}^{\prime} .
\end{aligned}
$$

Using (32) in (62) gives

$$
\begin{aligned}
0= & \tilde{V}_{, \alpha}+\left(\tau_{\alpha}^{c} \sigma_{c d} \rho^{d e}+\left(\zeta_{\alpha}^{e}+\tau_{\alpha}^{e}\right)\right)\left(-\rho_{a c} D^{c b}\left(u_{b}^{\mathrm{p}}-V_{, b}\right)-V_{, a}^{\prime}\right) \\
& -\left(A_{\alpha \gamma} B^{\gamma \delta} \zeta_{\delta}^{b}-\left(\zeta_{\alpha}^{c}+\tau_{\alpha}^{c}\right) \rho_{c d} D^{d b}\right)\left(u_{b}^{\mathrm{p}}-V_{, b}\right) \\
= & \tilde{V}_{, \alpha}-\tau_{\alpha}^{c} \sigma_{c d}\left(D^{d b}\left(u_{b}^{\mathrm{p}}-V_{, b}\right)-\rho^{d b} V_{, b}^{\prime}\right)-\left(\zeta_{\alpha}^{c}+\tau_{\alpha}^{c}\right) V_{, c}^{\prime}-A_{\alpha \gamma} B^{\gamma \delta} \zeta_{\delta}^{b}\left(u_{b}^{\mathrm{p}}-V_{, b}\right) \\
= & \tilde{V}_{, \alpha}-\left(\tau_{\alpha}^{c} \sigma_{c d} D^{d b}+A_{\alpha \gamma} B^{\gamma \delta} \zeta_{\delta}^{b}\right)\left(u_{b}^{\mathrm{p}}-V_{, b}\right)-\left(\tau_{\alpha}^{c} \sigma_{c d} \rho^{d b}+\zeta_{\alpha}^{b}+\tau_{\alpha}^{b}\right) V_{, b}^{\prime}
\end{aligned}
$$

Using the definition (31) of $D^{a b}$ and the identity (61), equation (63) simplifies to

$$
0=\tilde{V}_{, \alpha}-\left(\tau_{\alpha}^{c} \sigma_{c d} g^{d b}+\zeta_{\alpha}^{b}\right)\left(u_{b}^{\mathrm{p}}-V_{, b}\right)-\left(\tau_{\alpha}^{c} \sigma_{c d} \rho^{d b}+\zeta_{\alpha}^{b}+\tau_{\alpha}^{b}\right) V_{, b}^{\prime}
$$

Substituting the potential shaping portion of the control law

$$
u_{a}^{\mathrm{p}}=V_{, a}+D_{a c}\left(\tau_{\gamma}^{c} B^{\gamma \beta} V_{, \beta}-\rho^{c b} V_{, b}^{\prime}\right)
$$

from equation (33) gives

$$
0=\tilde{V}_{, \alpha}-\left(\tau_{\alpha}^{c} \sigma_{c d} g^{d b}+\zeta_{\alpha}^{b}\right) D_{b e}\left(\tau_{\gamma}^{e} B^{\gamma \beta} V_{, \beta}-\rho^{e f} V_{, f}^{\prime}\right)-\left(\tau_{\alpha}^{c} \sigma_{c d} \rho^{d b}+\zeta_{\alpha}^{b}+\zeta_{\alpha}^{b}\right) V_{, b}^{\prime} .
$$

Collecting coefficients of the partial derivatives of $V^{\prime}$ gives the potential matching condition (34).

\section{B Proof of Proposition 5.2}

Consider any compact, positively invariant set $\Omega \subset W$, where $W$ is given in (48), and let $E$ be the set of all points in $\Omega$ at which $\dot{E}_{\tau, \sigma, \rho}=0$ :

$$
E=\{(\phi, s, \dot{\phi}, \dot{s}) \in \Omega \mid 3 \dot{\phi}+\dot{s} \cos \phi=0\}
$$

Lasalle's invariance principle asserts that every trajectory starting in $\Omega$ approaches the largest invariant set $M$ contained in $E$ as $T \rightarrow \infty$. If the set $M$ contains only the equilibrium at the origin, it follows that the equilibrium is asymptotically stable. The set $\Omega$ provides an estimate of the region of attraction. 
By definition, $E_{\tau, \sigma, \rho}$ is constant along trajectories contained in $M$. Also, since

$$
\begin{aligned}
\frac{d}{d T} \varphi(\phi, s) & =\frac{d}{d T}\left(s+6 \operatorname{arctanh}\left(\tan \left(\frac{\phi}{2}\right)\right)\right) \\
& =\sec \phi(3 \dot{\phi}+\dot{s} \cos \phi),
\end{aligned}
$$

$\varphi(\phi, s)$ is constant along trajectories contained in $M$. Noting that $\varsigma$, given in (46), is zero within the set $M$, the Euler-Lagrange equations restricted to this set are

$$
\begin{aligned}
\frac{d}{d T} \frac{\partial L_{\tau, \sigma, \rho}}{\partial \dot{\phi}} & =-\left(2 \tan \phi \sec ^{2} \phi+3 \kappa \varphi \sec \phi\right) \\
\frac{d}{d T} \frac{\partial L_{\tau, \sigma, \rho}}{\partial \dot{s}} & =-\kappa \varphi .
\end{aligned}
$$

Because $\varphi$ is constant, (67) implies that

$$
\frac{\partial L_{\tau, \sigma, \rho}}{\partial \dot{s}}=c_{1} T+c_{2}
$$

where $c_{1}=-\kappa \varphi$ and $c_{2}$ are constants. But, because the equilibrium is stable, trajectories in $\Omega$ are bounded. This implies that $c_{1}=0$ and $c_{2}=0$. Thus, $\varphi(\phi, s)=0$ and

$$
\frac{\partial L_{\tau, \sigma, \rho}}{\partial \dot{s}}=0 .
$$

Restricted to the set $M$, the momenta conjugate to $\phi$ and $s$ are

$$
\begin{aligned}
\frac{\partial L_{\tau, \sigma, \rho}}{\partial \dot{\phi}} & =\frac{2}{3} \sec ^{2} \phi \dot{s} \\
\frac{\partial L_{\tau, \sigma, \rho}}{\partial \dot{s}} & =\frac{2}{3} \sec \phi \dot{s} .
\end{aligned}
$$

It follows from (68) that $\dot{s}=0$, since $\sec \phi \neq 0$ in $W$. From (69), we see that

$$
\frac{\partial L_{\tau, \sigma, \rho}}{\partial \dot{\phi}}=0
$$

From (66), it follows that $\phi=0$. Finally, since $\varphi(\phi, s)=0$ and $\phi=0$, it follows that $s=0$ as well. Thus, for any $\Omega \subset W$, the set $M$ contains only the origin. Asymptotic stability within the set $W$ follows from Lasalle's invariance principle.

\section{Proof of Proposition 5.3}

We note that the coefficients $p_{0}$ through $p_{3}$ given in (57) are linear in $k_{\text {diss }}$ and $\kappa$. For convenience, we let

$$
\begin{array}{ll}
p_{0}=-b_{0} \kappa & p_{2}=a_{2}-b_{2} \kappa+c_{2} k_{\text {diss }} \\
p_{1}=a_{1}-b_{1} \kappa+c_{1} k_{\text {diss }} & p_{3}=a_{3}+c_{3} k_{\text {diss }}
\end{array}
$$

where the coefficients $a_{i}, b_{i}$ and $c_{i}$ are defined by comparing with (57). Referring to (58), we have

$$
\begin{array}{ll}
p_{0}>0 \Leftrightarrow-b_{0} \kappa>0 & p_{2}>0 \Leftrightarrow k_{\text {diss }}>\frac{b_{2}}{c_{2}} \kappa-\frac{a_{2}}{c_{2}} \\
p_{1}>0 \Leftrightarrow k_{\text {diss }}<\frac{b_{1}}{c_{1}} \kappa-\frac{a_{1}}{c_{1}} & p_{3}>0 \Leftrightarrow k_{\text {diss }}<-\frac{a_{3}}{c_{3}}
\end{array}
$$


The condition on $p_{0}$ implies that $\kappa>0$, since $b_{0}=-b<0$. The lines (in the $\kappa$ - $k_{\text {diss }}$ plane)

$$
k_{\mathrm{diss}}=-\frac{a_{3}}{c_{3}}, \quad k_{\mathrm{diss}}=\frac{b_{2}}{c_{2}} \kappa-\frac{a_{2}}{c_{2}}, \quad k_{\mathrm{diss}}=\frac{b_{1}}{c_{1}} \kappa-\frac{a_{1}}{c_{1}} \text { and } \kappa=0
$$

are boundaries of stability. It can be checked that $-\frac{a_{2}}{c_{2}}<-\frac{a_{1}}{c_{1}}<0$ and $\frac{b_{2}}{c_{2}}<\frac{b_{1}}{c_{1}}<0$ for $0<d_{\phi}<\sqrt{2}$. The two lines $p_{1}=0$ and $p_{2}=0$ have negative slopes and negative $k_{\mathrm{diss}}$-axis intercepts. Moreover, the line $p_{2}=0$ is steeper than $p_{1}=0$ when $d_{\phi}<\sqrt{2}$ and the $k_{\text {diss }}$-axis intercept of $p_{2}=0$ is greater in magnitude than that of $p_{1}=0$. Assume that $d_{s}>-\gamma d_{\phi}$, so that the line $p_{3}=0$ is above the $\kappa$ axis. See Figure 9 .

Next, we need $\delta>0$ where

$$
\delta=p_{2} p_{3}-p_{1}
$$

We make the following observations:

- By the definition (73) of $\delta$, we see that

$$
p_{2}=p_{1}=0 \Rightarrow \delta=0 \text { and } p_{3}=p_{1}=0 \Rightarrow \delta=0 .
$$

Thus the curve $\delta=0$ passes through the intersection of $p_{1}=0$ with $p_{2}=0$ and also through the intersection of $p_{1}=0$ with $p_{3}=0$.

- Using (71) and (73), we have

$$
\begin{aligned}
\delta=0 & \Rightarrow\left(a_{3}+c_{3} k_{\mathrm{diss}}\right)\left(a_{2}-b_{2} \kappa+c_{2} k_{\mathrm{diss}}\right)-\left(a_{1}-b_{1} \kappa+c_{1} k_{\mathrm{diss}}\right)=0 \\
& \Rightarrow \kappa=\frac{\left(a_{3}+c_{3} k_{\mathrm{diss}}\right)\left(a_{2}+c_{2} k_{\mathrm{diss}}\right)-\left(a_{1}+c_{1} k_{\mathrm{diss}}\right)}{b_{2}\left(a_{3}+c_{3} k_{\mathrm{diss}}\right)-b_{1}} .
\end{aligned}
$$

It can be seen that

$$
\kappa \rightarrow \pm \infty \quad \text { as } \quad k_{\text {diss }} \rightarrow-\frac{a_{3}}{c_{3}}+\frac{b_{1}}{b_{2} c_{3}}
$$

Thus the line $k_{\text {diss }}=-\frac{a_{3}}{c_{3}}+\frac{b_{1}}{b_{2} c_{3}}$ is an asymptote for $\delta=0$. Also $\frac{b_{1}}{b_{2} c_{3}}=\frac{d_{\phi}}{4 c}>0$. This implies that the asymptote lies above the line $p_{3}=0$. From (74), we see that

$$
\kappa \rightarrow \frac{c_{2} k_{\text {diss }}}{b_{2}}+\frac{a_{2}}{b_{2}}+\frac{a_{3} c_{2}-c_{1}}{c_{3} b_{2}} \text { as } k_{\text {diss }} \rightarrow \pm \infty .
$$

Now, $p_{2}=0$ has the form

$$
k=\frac{c_{2} k_{\mathrm{diss}}}{b_{2}}+\frac{a_{2}}{b_{2}}
$$

This means that the asymptote given by (76) is parallel to $p_{2}=0$ and to the right since

$$
\frac{a_{3} c_{2}-c_{1}}{c_{3} b_{2}}=\frac{\left(\gamma d_{\phi}+d_{s}\right) d_{\phi}}{4 b(\gamma-1)}+\frac{1}{4 b}>0
$$

since $d_{s}>-\gamma d_{\phi}$.

The observations made so far are illustrated in Figure 9. The asymptotes given in (75) and (76) are shown by dashed lines. There are two possible cases. In the first case, shown in Figure 9(a), the point of intersection of the lines $p_{1}=0$ and $p_{2}=0$ lies below the point of intersection of $p_{1}=0$ and $p_{3}=0$. In the second case, shown in Figure 9(b), the point of intersection lies above that of $p_{1}=0$ and $p_{3}=0$. Since $\delta=0$ is quadratic in $k_{\text {diss }}$ and linear in $\kappa$, it cannot intersect $p_{1}=0$ or $p_{2}=0$ at points other than those shown by the filled circles. Thus, the possible branches of $\delta=0$ must be as shown in Figure 9, for the cases considered above. 
Figure 9: Preliminary boundaries for stabilizing control parameter values. Hashes denote regions where the conditions for exponential stability are violated.

The above observations help us to determine the possible regions of stabilizing control parameter values. At the origin,

$$
\delta=p_{3} p_{2}-p_{1}=a_{3} a_{2}-a_{1}>0 \text { since } a_{3}, a_{2}>0>a_{1}
$$

Thus, by continuity of $\delta$ in $\kappa$ and $k_{\text {diss }}$, the possible region of stabilizing control parameter values $(\delta>0)$ lies between the curves for $\delta=0$ in one case (Figure 9(a)) and does not lie between the curves in the other case (Figure 9(b)).

Next, we need

$$
\delta_{1}=\delta p_{1}-p_{3}^{2} p_{0}>0 .
$$

Thus $\delta_{1}=0$ forms another boundary on the range of stabilizing parameter values.

- Note that

$$
\begin{array}{lll}
\delta_{1}=0 & \text { when } & \delta=0 \text { and } p_{3}=0, \\
& \text { and when } & \delta=0 \text { and } p_{0}=0, \\
& \text { and when } & p_{1}=0 \text { and } p_{3}=0, \\
& \text { and when } & p_{1}=0 \text { and } p_{0}=0 .
\end{array}
$$

Thus the curve $\delta_{1}=0$ passes through the intersections of $\delta=0$ with $p_{0}=0$ and $p_{3}=0$ and also through the intersections of $p_{1}=0$ with $p_{0}=0$ and $p_{3}=0$.

- One can check that

$$
\begin{aligned}
0=\delta_{1}= & \left(\left(a_{3}+c_{3} k_{\text {diss }}\right)\left(a_{2}-b_{2} \kappa+c_{2} k_{\text {diss }}\right)-\left(a_{1}-b_{1} \kappa+c_{1} k_{\text {diss }}\right)\right)\left(a_{1}-b_{1} \kappa+c_{1} k_{\text {diss }}\right) \\
& +\left(a_{3}+c_{3} k_{\text {diss }}\right)^{2} b_{0} \kappa \\
\Rightarrow & 0=\left(a_{3}+c_{3} k_{\text {diss }}\right) b_{2}-b_{1} \text { for large } \kappa \\
\Rightarrow & k_{\text {diss }}=-\frac{a_{3}}{c_{3}}+\frac{b_{1}}{b_{2} c_{3}} \text { for large } \kappa .
\end{aligned}
$$


Thus $k_{\text {diss }}=-\frac{a_{3}}{c_{3}}+\frac{b_{1}}{b_{2} c_{3}}$ is an asymptote for $\delta_{1}=0$.

- We note that $\delta_{1}=0$ is quadratic in $\kappa$ and cubic in $k_{\text {diss }}$. This means that for a given $k_{\text {diss }}$, there can be two roots for $\kappa$ (including repeated roots) or none. Likewise, for a given $\kappa$, there can be three roots for $k_{\text {diss }}$ (including repeated roots) or one.

The above observations, along with an examination of Figure 9, give a clear idea about the nature of $\delta_{1}=0$. This is schematically shown in Figure 10 where $\delta_{1}=0$ is sketched along with the other curves. Figure 10(a) corresponds to the case discussed in Figure 9(a) and Figure 10(b) corresponds to the case discussed in Figure 9(b).

Figure 10: Sketch showing the region of stabilizing control parameter values (in gray).

The crucial observation is that there is some part of $\delta_{1}=0$ that lies in the fourth quadrant. At the origin $\left(\kappa=k_{\text {diss }}=0\right)$,

$$
\delta_{1}=\left(a_{3} a_{2}-a_{1}\right) a_{1}<0,
$$

since $a_{3}, a_{2}>0>a_{1}$. Thus, by the continuity of $\delta_{1}$ in $\kappa$ and $k_{\text {diss }}, \delta_{1}>0$ inside the $\delta_{1}=0$ loop as shown in Figure 10. The region of stabilizing control parameter values is shown in gray in Figure 10. This completes the proof. 


\section{References}

[1] Acosta JÁ, Ortega R, Astolfi A. Interconnection and damping assignment passivity-based control of mechanical systems with underactuation degree one. Proc American Control Conf. Boston, MA. 2004, pp 3029-3032.

[2] Auckly D, Kapitanski L, White W. (2000), Control of nonlinear underactuated systems. Comm Pure Appl Math 2000; 53: 354-369.

[3] Auckly D, Kapitanski L. On the $\lambda$-equations for matching control laws. SIAM J Control Optim 2002; 41(5): 1372-1388.

[4] Blankenstein G, Ortega R, van der Schaft A. The matching conditions of controlled Lagrangians and IDA-passivity based control. Int J Control. 2002; 75(9): 645-665.

[5] Bloch AM. Nonholonomic mechanics and control. Springer-Verlag, New York, NY 2003.

[6] Bloch AM, Chang DE, Leonard NE, Marsden JE. Controlled Lagrangians and the stabilization of mechanical systems II: Potential shaping. IEEE Trans Automatic Control 2001; 46(10): 1556-1571.

[7] Bloch AM, Krishnaprasad PS, Marsden JE, Ratiu, TS. Dissipation induced instabilities. Ann Inst H Poincaré, Analyse Nonlinéaire 1994; 11: 37-90.

[8] Bloch AM, Krishnaprasad PS, Marsden JE, Sánchez de Alvarez G. Stabilization of rigid body dynamics by internal and external torques. Automatica 1992; 28(4): 745-756.

[9] Bloch AM, Leonard NE, Marsden JE. Stabilization of mechanical systems using controlled Lagrangians. Proc IEEE Conf Decision and Control. San Diego, CA. 1997, pp 2356-2361.

[10] Bloch AM, Leonard NE, Marsden JE. Matching and stabilization by the method of controlled Lagrangians. Proc IEEE Conf Decision and Control. Tampa, FL. 1998, pp 1446-1451.

[11] Bloch AM, Leonard NE, Marsden JE. Potential shaping and the method of controlled Lagrangians. Proc IEEE Conf Decision and Control. Phoenix, AZ. 1999, pp 1653-1657.

[12] Bloch AM, Leonard NE, Marsden JE. Stabilization of the pendulum on a rotor arm by the method of controlled Lagrangians. Proc Int Conf Robotics and Automation. Detroit, MI. 1999, pp 500-505.

[13] Bloch AM, Leonard NE, Marsden JE. Controlled Lagrangians and the stabilization of mechanical systems I: The first matching theorem. IEEE Trans Automatic Control 2000; 45(12): 2253-2270.

[14] Bloch AM, Leonard NE, Marsden JE. Controlled Lagrangians and the stabilization of Euler-Poincaré mechanical systems. Int J Robust and Nonlinear Control (Special Issue on Control of Oscillatory Systems) 2001; 11(3): 191-214.

[15] Branicky MS. Multiple Lyapunov functions and other analysis tools for switched and hybrid systems. IEEE Trans Automatic Control 1998; 43(4): 475-482.

[16] Bullo F, Lewis AD. Geometric control of mechanical systems. Springer-Verlag, New York, NY 2004. (To appear.)

[17] Chang DE. Controlled Lagrangian and Hamiltonian systems. PhD Thesis, California Institute of Technology.

[18] Chang DE, Bloch AM, Leonard NE, Marsden JE, Woolsey CA. The equivalence of controlled Lagrangian and controlled Hamiltonian systems for simple mechanical systems. ESAIM: Control, Optimisation, and Calculus of Variations (Special Issue Dedicated to JL Lions) 2002; 8: 393-422. 
[19] Gómez-Estern F, van der Schaft A. Physical damping in IDA-PBC controlled underactuated mechanical systems. European J Control 2004. (In review.)

[20] Hamberg J. General matching conditions in the theory of controlled Lagrangians. Proc IEEE Conf. Decision and Control. Phoenix, AZ. 1999, pp 2519-2523.

[21] Khalil, HK. Nonlinear systems. $3^{\text {rd }}$ edn. Prentice Hall, Upper Saddle River, NJ 2002.

[22] Marsden JE, Ratiu TS. Introduction to mechanics and symmetry. Springer-Verlag, New York, NY 1994.

[23] Marsden JE. Lectures on mechanics. Cambridge University Press, New York, NY 1992.

[24] Murray RM, Li Z, Sastry SS. A mathematical introduction to robotic manipulation. CRC Press, Boca Raton, FL 1994.

[25] Ortega R, Spong MW, Gómez-Estern F, Blankenstein G. Stabilization of a class of underactuated mechanical systems via interconnection and damping assignment. IEEE Trans Automatic Control 2002; 47(8): 1218-1233.

[26] Reddy CK, Whitacre WW, Woolsey CA. Controlled Lagrangians with gyroscopic forcing: An experimental application. Proc American Control Conf. Boston, MA. 2004, pp 511-516.

[27] Sánchez de Alvarez G. Geometric methods of classical mechanics applied to control theory. PhD Thesis. University of California, Berkeley. 1986.

[28] Wang LS, Krishnaprasad PS. Gyroscopic control and stabilization. J Nonlinear Sci 1992; 2: 367-415.

[29] Woolsey CA, Bloch AM, Leonard NE, Marsden JE. Physical dissipation and the method of controlled Lagrangians. Proc European Control Conf. Porto, Portugal. 2001, pp 2570-2575.

[30] Woolsey CA, Bloch AM, Leonard NE, Marsden JE. Dissipation and controlled Euler-Poincaré systems. Proc IEEE Conf Decision and Control. Orlando, FL. 2001, pp 3378-3383. 